\title{
Numerical simulation of stratified flow around a tall building of a complex shape
}

\author{
Michał Korycki ${ }^{1}$ Lech Lobocki ${ }^{2}$ Andrzej Wyszogrodzki ${ }^{3}$
}

Received: 28 September 2015/Accepted: 12 July 2016/Published online: 23 July 2016

(C) The Author(s) 2016. This article is published with open access at Springerlink.com

\begin{abstract}
Results of large-eddy simulations of stably stratified atmospheric flow around an isolated, complex-shaped tall building are presented. The study focuses on the identification of flow structures in the building wake in high and low Froude number regimes. A qualitative comparison of results with available literature data and existing theories is presented. In addition to flow structures identified in earlier studies such as the horseshoe and recirculation eddy vortices, we analyze a stationary disturbance akin to mountain gravity wave, and a complex vortex structure associated with this wave, consisting of multiple symmetric pairs of vortices. The Froude number appears to be the principal parameter controlling the structure of the wake, waves and vortex pattern.
\end{abstract}

Keywords Urban fluid mechanics - Stably Stratified flows · Building wake · Building aerodynamics

\section{Introduction}

Studies of flow around buildings began in the middle of the past century. In an early review, Hunt [27] listed five areas of practical interest: wind regime change at a pedestrian level, heating and ventilation of buildings, pollutants' dispersion from and around buildings, wind noise, and the safety of aircraft take-off and landing at cities' airports.

Michał Korycki

michal.korycki@ncbj.gov.pl

1 National Centre for Nuclear Research, Otwock, Poland

2 Faculty of Building Service, Hydrotechnics and Environmental Engineering, Warsaw University of Technology, Warsaw, Poland

3 Institute of Meteorology and Water Management, Warsaw, Poland 
Subsequently, these studies expanded into other areas, such as wind loads on structures or aeroelasticity of tall buildings $[8,14]$. At present, circumfluence of buildings is one of the principal branches of urban fluid mechanics [17].

Since the creation of the world's first meteorological wind tunnel capable of simulating flow in a thermally stratified boundary layer $[7,54]$ and the pioneering wind-tunnel simulation of flow around a group of buildings [29], wind-tunnel experiments became frequently used to investigate flows within urban fabric. In the 1970s, analytical theories of wake development were presented, e.g. [26]; soon, these were complemented and eventually superseded by advances in computational fluid dynamics (CFD). Notably, many of the early CFD studies were devoted to validation of CFD methods and models, and a vast majority of such efforts focused on the comparison of their results to those obtained from wind-tunnel experiments. Several reviews of these works and findings are available, e.g. $[44,45,53,80]$. In spite of the CFD capabilities, experiments were mostly focused on idealized, simplified problems such as a steady-state adiabatic flow around a cubic building under statically neutral conditions. Nevertheless, the growing confidence in CFD results eventually paved the way for CFD application as an independent design tool in engineering $[74,81]$. Further, detailed measurements of the flow structure and pollutant dispersion within the urban fabric became available (e.g. [3, 4, 24, 58]), and numerical models encompassing city blocks or quarters were built (e.g. [9, 18, 20, 23, 52])

Wind-tunnel and computational studies identified several salient structures in building wakes (see e.g. [31, 53] for reviews). Many studies report a horseshoe-shaped vortex with a slightly inclined symmetry axis that surrounds the building, stretching behind into the wake (e.g. $[5,86])$. This is often accompanied by an arc-shaped vortex developing behind the obstacle. Usually, a recirculation zone develops behind the building, along the axis of the wake. Several smaller recirculation cells on the windward side of the building, near the ground level, can also be present, as well as a cell developing over a flat roof top [5]. These features are associated with a separation of the flow and a reattachment pattern at the ground or at the building surface.

The influence of buildings on the structure of the flow within the urban canopy layer is not unambiguous, particularly with regard to the pedestrian-level wind field. On a cityscale, drag forces caused by the impingement of the flow on building walls deflect the areamean wind and decrease its speed. However, in smaller scales, the airflow may actually accelerate in gaps between buildings. Murakami et al. [46] studied amplification of pedestrian-level wind speed by tall buildings caused by momentum transport from upper part of the canopy, both by means of numerical modelling and by wind-tunnel studies. They found that an isolated high-rise building may amplify the pedestrian-level wind speed by as much as 50-100\% and that this effect can be even larger in vicinities occupied by low buildings.

Although the atmospheric stability is a key factor in atmospheric dispersion, it has received much less attention in past studies. This apparent lack of interest may be partially explained by the abundance of mechanically generated turbulence which reduces the relative role of buoyancy effects, and by the reduction of surface cooling during nighttime by the urban heat storage [6]. Notwithstanding, numerous studies (e.g. [10, 32, 33, 38]) have thoroughly documented severe wintertime air pollution episodes associated with temperature inversions. These conditions may deserve special attention, at least from the city ventillation perspective. Only a decade ago, Britter and Hanna [6] placed the stratification at the first position on their list of "the most pressing problems" in urban fluid mechanics; since this time, many questions still remain open. 
By the end of the twentieth century, a few wind tunnels capable of simulating thermal stratification effects on the boundary-layer flow were already in place (e.g. $[49,50,54,56,60])$, with some of them capable of reproducing flow structures as complex as sea-breeze circulation [40] or as a convective boundary layer capped with an inversion [16]. While some of the wind-tunnel studies addressed stratified flows around buildings (e.g. [31]), it was left for numerical modelling to complement the limited capabilities of wind tunnels by simulations of more complex environmental conditions. The tremendous advance in computational fluid dynamics (CFD) in the last few decades has greatly extended our research capabilities, particularly due to large-eddy simulation technique, emerging as possibly the most accurate method in urban fluid mechanics [20, 44, 45, 80]. However, among a large number of computational studies reported to date, only a small fraction avoided a typical scenario of steady-state, neutrally stratified flow around simplyshaped buildings. Studies of stably stratified urban flows, either numerical or experimental represent a narrow class, and most of them focus on pollutant dispersion in street canyons (e.g. [34, 82]). To this date, flow around tall buildings under stable conditions has been addressed in just a few studies [30, 31, 59, 90].

An important question regarding the atmospheric flow over an isolated obstacle has been phrased by Sheppard [63]: "Under what conditions will an airstream rise over a mountain range?" (as opposed to flowing around). Although the scales of mountain and urban flows are different, the mechanisms may be similar, at least qualitatively. In view of the scope of this paper, two linear theories should be mentioned: potential-flow solutions, applicable to high Froude number flows $[15,25,36]$ and the solution derived from linear gravity-wave theory by Smith [64] for low Froude number flows. While these two theories apparently lack a common overlapping validity region, the numerical study by Smolarkiewicz and Rotunno [70] provides a solution for the mixed moderateto-low Froude number regime that can be useful for a quantitative comparison.

In the following, we shall present results of large-eddy simulations (LES) of stably stratified flow around an isolated tall building. To identify the thermal stratification effects alone, no influence on the undisturbed wind profile is assumed and a constant vertical ambient temperature gradient is set within the entire simulation domain. The simulations were performed using the EULAG code, a wideband hydrodynamic solver designed for geophysical flows [55]. This code was successfully used in diverse applications, ranging from mechanics of brain injuries [13] to various meteorological studies (e.g. [22]) and to solar convection [19, 65]. It was validated in rotating stratified flows with scales ranging from laboratory experiments [85] to global circulations [69], including orographic flows in the atmospheric boundary layer [70] and in the ocean [83]. In the context of urban flows, EULAG was applied to simulations of stratified flows over complex isolated buildings (e.g. Pentagon [71]) and realistic large urban environments (e.g. Oklahoma City [88]) and served as a small-scale computation kernel in the integrated mesoscale/urban modeling system built by Chen et al. [9]. At present, works on utilizing EULAG techniques in the world's leading numerical weather prediction systems - the Integrated Forecast System (IFS) at the European Center for Medium-Range Weather Forecast [51] and the Consortium's for Small-scale Modelling Local Model (COSMO-LM) [57]—are in progress. 


\section{Numerical experiments}

\subsection{Fluid solver description}

The EULAG model used in this study allows for several optional formulations of the predictive equation system [69]. The particular version adopted here solves a perturbation form of conservation equations in a nonhydrostatic anelastic approximation. Basic thermodynamic variables are scaled with a set of reference state $(\bar{\phi})$ variables, and decomposed into a hydrostatically-balanced, vertically-dependent "environmental" equilibrium state $\left(\phi_{e}\right)$ and its perturbances (departures) $\left(\phi^{\prime}\right)[11]$.

Following [71], the building impact on the flow is simulated using the immersed boundary method (IMB). With this approach, the anelastic system of equations is written as:

$$
\begin{gathered}
\frac{d \mathbf{v}}{d t}=-\nabla \pi^{\prime}-\mathbf{g} \frac{\theta^{\prime}}{\bar{\theta}}-\beta\left(\mathbf{v}-\mathbf{v}_{\mathbf{b}}\right)-\alpha_{\mathbf{m}} \mathbf{v}^{\prime}+\mathbf{D}_{\mathbf{v}} \\
\frac{d \theta^{\prime}}{d t}=-\mathbf{v} \cdot \nabla \theta_{e}-\beta\left(\theta-\theta_{b}\right)-\alpha_{h} \theta^{\prime}+D_{\theta} \\
\frac{d e}{d t}=S_{e}-\beta\left(e-e_{b}\right) \\
\nabla(\bar{\rho} \cdot \mathbf{v})=0
\end{gathered}
$$

where $\mathbf{v}$ is the three-dimensional velocity vector; $\pi^{\prime}=p^{\prime} / \bar{\rho}$ is the normalized pressure perturbation (derived applying the anelastic continuity Eq. 4); $\theta$ is the potential temperature, $\rho$ denotes the density of air, $g$ is the gravity, and the total derivative is

$$
\frac{d \phi}{d t} \equiv \frac{\partial \phi}{\partial t}+\frac{1}{\bar{\rho}} \nabla \cdot(\bar{\rho} \mathbf{v} \phi) .
$$

The overbar denotes a dry reference state (i.e. $\bar{\rho}$ and $\bar{\theta}$ ), whereas primes denote deviations from a hydrostatically balanced "environmental" state $\left(\mathbf{v}_{e}, \theta_{e}\right) . \beta$ is a relaxation constant which will be explained later (Sect. 2.2). The "environmental" state is a priori assumed state satisfying model equations, and in general may represent the time dependent undisturbed flow as e.g. oceanic tidal motion [83], or geostrophically and thermally balanced large-scale flow $([22,66,69])$. The particularly chosen set of approximations may represent either deep (anelastic, pseudo-incompressible) or shallow (Boussinesq limit, constant reference atmospheric state) motion approximations-further details on these perturbation equations are given in [35]. The use of ambient state in the perturbation form of governing equations allows for a simplification of the initial state, for the design of inflow boundary conditions and for enhancing accuracy of finite-precision arithmetics.

Terms $D_{\mathbf{v}}$ and $D_{\theta}$ represent turbulent transport of momentum and heat

$$
\begin{gathered}
D_{\mathbf{v}}=\tau_{i j}=-K_{m}\left(\frac{\partial u_{i}}{\partial x_{j}}+\frac{\partial u_{j}}{\partial x_{i}}\right) \\
D_{\theta}=-K_{h}\left(\frac{\partial \theta}{\partial x_{j}}\right)
\end{gathered}
$$


where $K_{m}$ and $K_{h}$ are eddy viscosity and eddy diffusivity coefficients, respectively which depend on the subgrid turbulent kinetic energy (TKE), mixing length $\lambda=\min \left(\Delta, c_{L} Z\right)$, effective grid size $\Delta=(d x+d y+d z) / 3$ and the Prandtl number $\operatorname{Pr}=c_{m} / c_{h}$,

$$
\begin{gathered}
K_{m}=c_{m} \lambda e^{1 / 2} \\
K_{h}=K_{m} / P r
\end{gathered}
$$

Here, we used $c_{m}=0.0856, c_{h}=0.204, c_{L}=0.845$. The parametrization of turbulent transport is based upon the budget (3) of the subgrid-scale TKE, where $S_{e}$ denotes sources and sinks, such as the TKE production by shear, buoyancy, turbulent transport and dissipation, following [39] and [73]:

$$
S_{e}=-\tau_{i j} \frac{\partial u_{i}}{\partial x_{j}}+\frac{g}{\bar{\theta}} \frac{\partial \theta}{\partial z}+c_{e} \frac{\partial}{\partial x_{j}}\left(K_{m} \frac{\partial}{\partial x_{j}} e\right)-c_{\epsilon} e^{3 / 2} / \lambda
$$

with coefficients $c_{e}=0.845$, and $c_{\epsilon}=2.0$. Coefficients $\alpha_{m}=1 / \tau_{m}[1 / s]$ and $\alpha_{h}=$ $1 / \tau_{h}[1 / s]$ are reciprocals of (long) time scales of the Rayleigh friction and Newtonian cooling, where $\tau_{m}$ and $\tau_{h}$ are the characteristic attenuation time scales with their values ranging typically from a few to tens of minutes and their damping factor decreasing linearly from one at the boundary to zero at a certain distance $L_{m, h}$ away from it, depending on the time scale of the perturbation approaching the boundary. The role of these coefficients is to attenuate solution (if required), to the prescribed ambient state in the vicinity of open model boundaries. In our case the solution is strongly determined by the inner domain forcing, hence the explicit attenuation to the external state is not feasible as it could affect the flow structures generated on the lee side of the building. Therefore, the Rayleigh damping is not applied in our experiments.

All variables are co-located on a regular, logically rectangular mesh. The finite-difference approximations to the temporal integrals in Eq. (1-3) represent the second-order accurate nonoscillatory forward in time (NFT) transport operator in the Eulerian flux-form. The generalized transport scheme used here, the Multidimensional Positive Definite Advection Transport Algorithm (MPDATA) is widely documented in the literature (see e.g. the review [67]). Equations (1-4) represent a system which is implicit with respect to all resolved variables and which results in an elliptic equation for the pressure $\pi^{\prime}$, subject to the integrability condition imposed on velocity components normal to the external boundary. The resulting boundary value problem is solved-with an accuracy to a judiciously specified threshold-using a preconditioned nonsymmetric Krylov subspace solver known as the Generalized Conjugate Residual (GCR) algorithm [68].

\subsection{Immersed boundary parametrization}

In the IMB approach adopted here (see Appendix $C$ in [71] for more details on the IMB scheme), building effects are simulated by entering source terms into the predictive equations, and enforcing relaxation to a prescribed state within the volume occupied by the building. The corresponding flow variables describing this state are denoted by subscript $b\left(\mathbf{v}_{b} \equiv 0, e_{b} \equiv 0, \theta_{b}\right)$. The implicit time discretization of the repelling forces (i.e., a variant of the feedback forcing [43]) admits rapid attenuation of the flow to stagnation (within solid structures) in $O(\delta t)$ time comparable to the time step $\delta t$ of the fluid model (see [71] for an exposition). 
The coefficient $\beta$ is the inverse time scale of the repelling body force introduced to mimic no-slip boundary conditions at the building walls. In the simulations we set $\beta^{-1}=0.5 \delta t$. A shorter time scale of the forcing is still stable, however it may give an oscillatory solution within the building. This variant of the IMB is effective for simulating small scale transport and dispersion within intricate urban structures. Such approximate imposition of internal-boundary conditions offers relative computational simplicity and massively-parallel efficacy, compared to schemes with explicit specification of the internal boundaries.

The EULAG-IMB approach outlined above has been applied throughout a range of scales, including simulation of Darcy flows in microscopic porous media [72], idealized flows around complex isolated building structures [71] and realistic stratified atmospheric flows in large city environments [87, 88].

\subsection{Model configuration and background meteorological conditions}

Two basic approximation sets have been used: an anelastic system as described above, and a shallow-motion Boussinesq approximation that may be considered as a special case of Eqs. (1-4). In the latter, the reference-state density is taken as constant (and thereby drops out of the continuity Eq. 4), and the stratification is imposed by setting the gradient of the ambient potential temperature. As no discernible differences were found in temporally averaged results (as explained later), we shall skip the discussion of the choice of approximated equation system. The anelastic reference state is defined by a stability parameter $S$, such that

$$
\bar{\theta}(z)=\theta_{0} e^{S z}
$$

where $\theta$ is a potential temperature, $\mathrm{z}$ is the height above the terrain, and $\theta_{0}$ is the potential temperature at the ground surface. The overbars denote the reference state (i.e. $\bar{\rho}, \bar{\theta}$ ). For a sufficiently small range of heights,

$$
S=\frac{1}{\bar{\theta}} \frac{\partial \bar{\theta}}{\partial z}=\frac{N_{B V}^{2}}{g}
$$

where $N_{B V}$ is the Brunt-Väisälä frequency. For a convenient comparison with results of other studies, we shall also list the Froude number

$$
\mathrm{Fr}=\frac{U}{N_{B V} H}
$$

where $U$ is the characteristic undisturbed flow velocity, taken here as $3 \mathrm{~m} \mathrm{~s}^{-1}$, and $H$ is the obstacle height (here, $190 \mathrm{~m}$ ).

The building (see Fig. 1) - approximately $230 \mathrm{~m}$ tall (height without the spire $\mathrm{H}=190 \mathrm{~m}$ ) and $270 \mathrm{~m}$ wide at its base-is the tallest to date building in Poland, known as Pałac Kultury i Nauki (PKiN). For more details, see the official building website [77]. The building representation was built by a ray-tracing voxelization algorithm, using a digital 3-d city model [84], kindly provided by the City Hall of Warsaw. This database contained building data represented by organized sets of planes defining the exterior shell of a given building.

The model domain was set in a Cartesian coordinate frame, with its $X$ axis oriented parallel to the symmetry plane of the building (approximately eastward). The undisturbed 

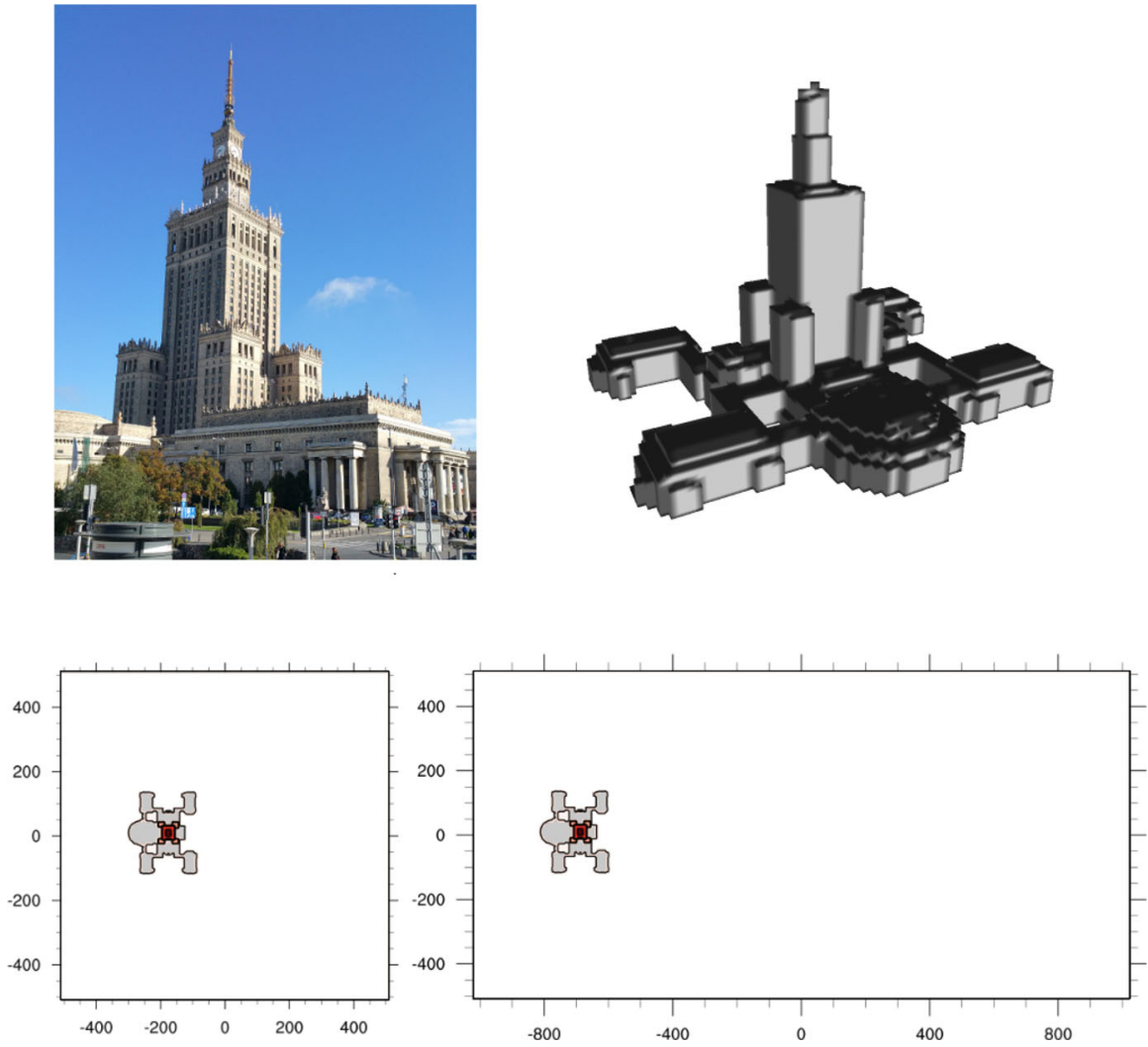

Fig. 1 PKiN (top) and grid configuration of base domain $256^{3}$ (lower left) and extended domain $512 \times$ $256 \times 256$ (lower right). The voxelized building model is rendered using the VAPOR visualization tool [12]

wind direction was assumed along this axis. The grid comprises either $256^{3}$ uniformly spaced nodes in the series of high Froude number experiments or $512 \times 256 \times 256$ in low Froude number range experiments. For the latter, we found it necessary to extend the domain, doubling the number of gridpoints along the undisturbed flow direction. In both series, the horizontal spacing was $4 \mathrm{~m}$ and the vertical was $2 \mathrm{~m}$. The model was run in its Eulerian and anelastic options using a $0.05 \mathrm{~s}$ time step while a larger value, $0.2 \mathrm{~s}$ was used later in the shallow-motion configuration.

In overall, we report results of thirteen simulations of idealized meteorological conditions and one case study based upon observed situation (Sect. 6). To facilitate comparison with other studies, simulation of flow around a cuboidal building was also included (Sect. 5). Idealized flow simulations described in Sects. 3 and 4 were carried out with different values of stability parameters summarized in Table 1 , and with the same undisturbed wind profile, namely, wind speed increasing linearly from $3 \mathrm{~m} \mathrm{~s}^{-1}$ at the lowest model level $(2 \mathrm{~m})$ with a vertical gradient of $0.002 \mathrm{~s}^{-1}$ and no turn in height. These undisturbed profiles were also used to form both the initial and the lateral boundary conditions as explained in the next subsection. 
Table 1 Numerical values of stability parameters used in simulations, and corresponding lengths of lee waves as calculated from Eq. 15. Symbols are explained in the text

\begin{tabular}{lrrlll}
\hline Case \# & \multicolumn{1}{l}{$S\left(\mathrm{~m}^{-1}\right)$} & $\frac{\partial \bar{\theta}}{\partial z}\left(\mathrm{~K} \mathrm{~m}^{-1}\right)$ & $N_{B V}\left(\mathrm{~s}^{-1}\right)$ & Fr & $\lambda_{x}(\mathrm{~m})$ \\
\hline 1 & $1 \times 10^{-6}$ & $2.88 \times 10^{-4}$ & 0.0031 & 5 & 6015 \\
2 & $1 \times 10^{-5}$ & $2.88 \times 10^{-3}$ & 0.0099 & 1.6 & 1902 \\
3 & $1.5 \times 10^{-5}$ & $4.32 \times 10^{-3}$ & 0.0121 & 1.3 & 1553 \\
4 & $2 \times 10^{-5}$ & $5.76 \times 10^{-3}$ & 0.0140 & 1.13 & 1345 \\
5 & $2.5 \times 10^{-5}$ & $7.2 \times 10^{-3}$ & 0.0157 & 1.01 & 1203 \\
6 & $3 \times 10^{-5}$ & $8.64 \times 10^{-3}$ & 0.0172 & 0.92 & 1098 \\
7 & $5 \times 10^{-5}$ & $1.44 \times 10^{-2}$ & 0.0221 & 0.71 & 851 \\
8 & $7.5 \times 10^{-5}$ & $2.16 \times 10^{-2}$ & 0.0271 & 0.58 & 695 \\
9 & $1 \times 10^{-4}$ & $2.88 \times 10^{-2}$ & 0.0313 & 0.50 & 602 \\
10 & $1.25 \times 10^{-4}$ & $3.6 \times 10^{-2}$ & 0.0350 & 0.45 & 538 \\
11 & $1.5 \times 10^{-4}$ & $4.32 \times 10^{-2}$ & 0.0383 & 0.41 & 491 \\
12 & $1.75 \times 10^{-4}$ & $5.04 \times 10^{-2}$ & 0.0414 & 0.38 & 455 \\
13 & $2 \times 10^{-4}$ & $5.76 \times 10^{-2}$ & 0.0443 & 0.36 & 425 \\
\hline
\end{tabular}

\subsection{Boundary conditions}

Thanks to the separation of a balanced "environmental" state in the EULAG model, boundary conditions only apply to the perturbation part of flow variables and do not need to provide forcing necessary to maintain the domain-scale equilibrium of forces and homogeneity in the background flow. A broader discussion of this feature is presented in Sect. 6, where a real meteorological situation is studied; with the highly idealized flow discussed in the next three sections, we adopted a very simple approach. Laminar flow $\left[u_{e}(z), 0\right]$ conditions were set at the inflow boundary. The outflow boundaries in the lateral and streamwise directions were open, with absorbing layers switched off. Free-slip type boundary conditions were imposed at the ground, and an impermeable rigid boundary with a wave absorber was set at the top of the computational domain. No-slip and partial-slip type boundary conditions at the ground surface were also tested for comparison. In the case of no-slip type conditions, the effects on salient flow structures turned out to be barely discernible, except for the immediate vicinity of the ground surface; with partial-slip type conditions imposed on the surface stress through assuming a fixed drag coefficient value $\left(C_{d}=0.01\right)$, we observed only minor quantitative differences in the peak values of the wind and temperature, without significant changes in major flow structures. A broader discussion of these results, possibly in a more general context, is postponed to a separate publication. As the IMB method relies on introducing reaction to flow impingement as source terms in momentum budget equations rather than on a formulation of boundary conditions, we also refrained from using additional stress parametrizations in the vicinity of walls, entirely relying on the LES and its subgrid turbulence scheme as transport mechanisms. The temperature of the building walls was subject to relaxation to a prescribed temperature of the building interior. These simplifications deserve subsequent studies, aiming at parametrization schemes specifically designed for use under various stability conditions with the IMB approach. 


\subsection{Averaging procedure}

Left-hand side panels of Fig. 2 display vertical velocity contours and streamlines plotted in two cross-sections at an arbitrarily taken moment (1200 s) in a moderately stable flow. In a horizontal $(X Y)$ cross-section located near the ground $(20 \mathrm{~m})$, a narrow band of ascending motion along the centerline of the wake is present. Interspersed filaments of ascending and descending air extend outward from the wake centerline in a V-shaped pattern. These filaments are footprints of vortex structures which travel with the mean flow. Farther from the wake centerline, at the lateral edges of the wake, vortex structures traveling with the flow form a pattern resembling the von Kármán's vortex path. This feature is even more evident in the vorticity patterns (not shown). While these transient structures appear to dominate the flow, their irregular, chaotic nature calls for some statistical approach to be applied. Postponing the discussion of numerical values of particular statistics to a subsequent publication, we shall focus on a qualitative discussion of mean flow structures and their dependence on stability.

To filter out transient features and to isolate salient forms of the mean flow, a simple temporal averaging procedure was applied. In our experiments, adaptation of the flow was apparent after some 7 mins (8400 steps) of the integration time in case of the $256^{3}$ domain, and after roughly 13 mins in the case of extended domain. While the flow still remained unsteady after this time, the flow structures may be analysed with a proper time averaging [28]. Specifically, the averaging involved 28,000 time steps after additional 8000 steps spent on the spin-up in our anelastic configuration, and $7000+4000$, correspondingly, in the case of the shallow-motion setup. These numbers roughly match recommendations [21], despite the different setup of experiments. In this study, we shall focus on a set of fields, obtained by averaging the numerical solutions point-by-point, throughout a 23-min
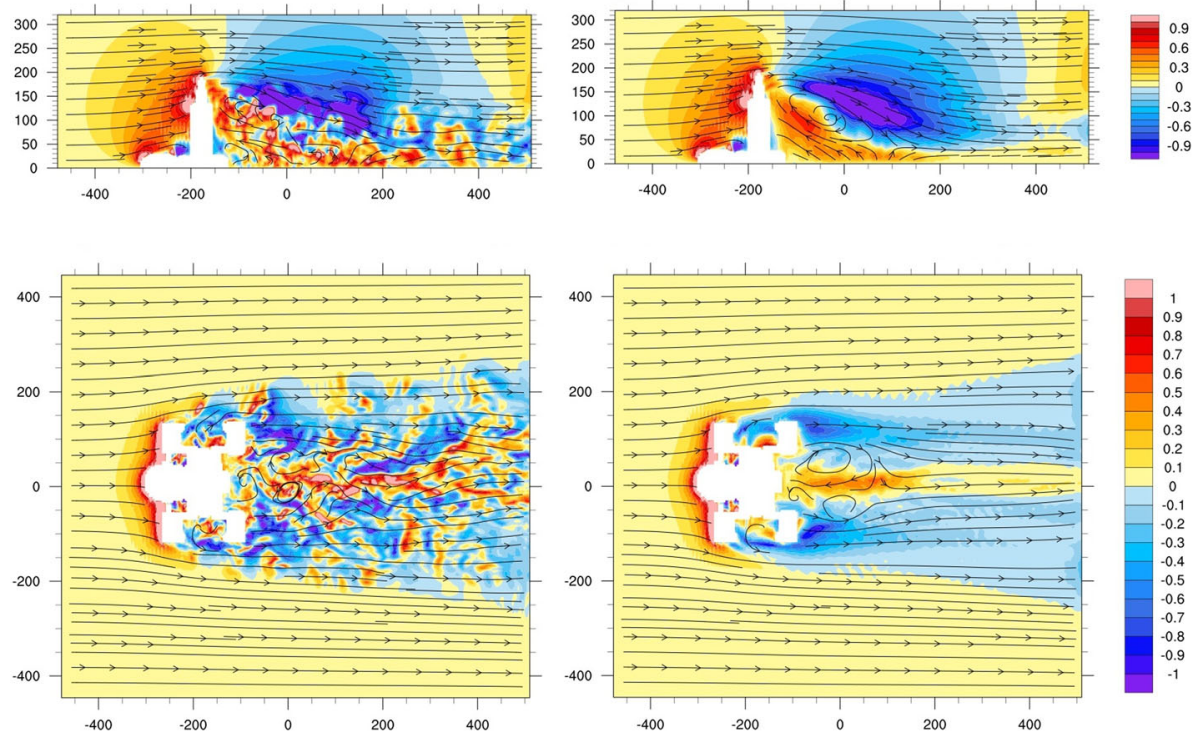

Fig. 2 Patterns of vertical velocity in $X Z$ (top) and $X Y$ (bottom) cross-sections: instantaneous values at $1200 \mathrm{~s}$ (left) and temporally averaged (right). This, and all the remaining plots were made using the NCL package [76] 
simulation period after the spin-up. Figure 2 illustrates the effects of such filtering. As can be seen, transient structures have been effectively eliminated revealing the existence of organized stationary motions, such as the recirculation vortex at the lee side of the building in the central vertical cross-section (upper right panel) or a pair of vortices rotating in a horizontal plane in the wake immediately behind the building (lower right panel). Overall, averaged solutions obtained by this LES procedure resemble solutions of Steady-state Reynolds Averaged Numerical Simulation (SRANS) models presented in the literature. In the following, we shall discuss the formation of salient structures of the mean flow under various stability conditions.

\section{Salient structures of the mean flow at high Froude numbers}

We shall begin with an examination of salient flow structures forming under neutral-tomoderate stability, in flows where the Froude number is larger than or approximately equal to unity.

The majority of simulations of stably stratified flows around buildings were carried out using the SRANS technique, and focused on idealized (typically, cuboid) building shapes (e.g. Zhang et al. [90]). In contrast, we have applied the LES approach to the flow around a realistic, complex-shape building. Hence, one may anticipate substantial differences in the near-building flow structures in comparison to those formerly reported. Figure 3 presents $X Z$ cross-sections of mean fields of: potential temperature departure $\theta^{\prime}$, vertical velocity $w$, pressure perturbation $p^{\prime}$, and subgrid turbulent kinetic energy $e$ for a moderately stable case.

The flow impinging on the windward face of the building is decelerated and its momentum is transformed in the lateral and vertical direction. As can be seen from Fig. 3, flow structures develop vertically both in the close vicinity of the building and in the farfield solution. Small recirculation zones appear over the lower rooftops at the windward base of the main vertical building part with a local maximum of the TKE. Along windward walls, updrafts arise causing the boundary layer separation. Maximum vertical velocity, over $1 \mathrm{~m} \mathrm{~s}^{-1}$ is observed near the top edge of the main vertical part of the building, near the viewing terrace at the 30-th floor (114 m a.g.1.). Interestingly, at the same height on the lee
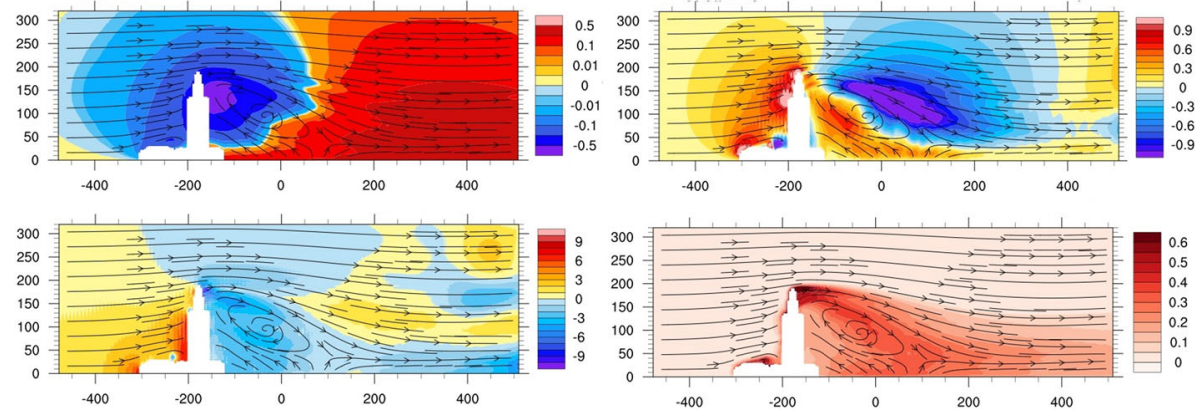

Fig. $3 X Z$ cross-section of the mean flow properties for a moderately stable case of $S=3 \times 10^{-5} \mathrm{~m}^{-1}$. XZplane streamlines are shown at a background of contour plots of: potential temperature departure (upper left); vertical velocity (upper right); pressure perturbation (lower left) and subgrid turbulent kinetic energy (lower right) 

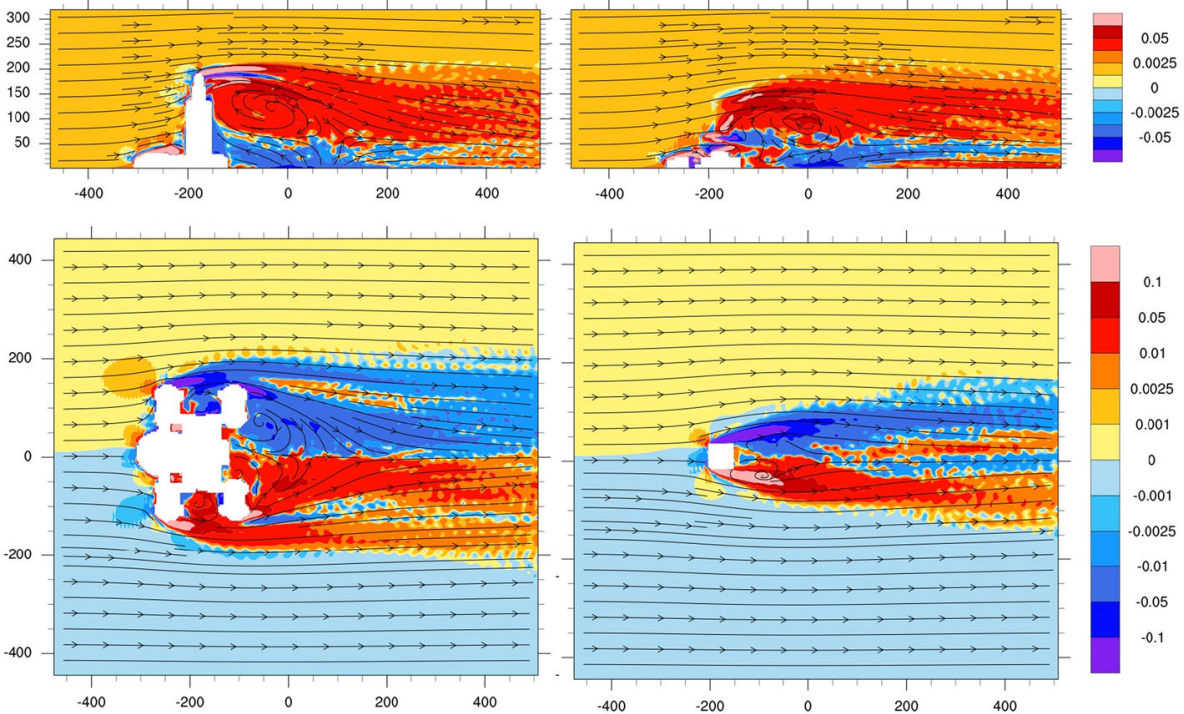

Fig. 4 Vorticity at neutral equilibrium. Upper panels $X Z$ cross-sections, at the symmetry plane (wake center, left) and at $y=-30 \mathrm{~m}$; lower panels $X Y$ cross-section at $z=10 \mathrm{~m}$ (left) and $z=122 \mathrm{~m}$ (right). Contour plots show averaged vorticity components perpendicular to the section plane, and the streamlines are calculated using $(x, z)$ and $(x, y)$ components, respectively

side of the building, an area with local minimum of the mean departure of potential temperature forms, which is associated with an increase of local static stability. Also on the lee side, a well-developed recirculation zone is present with its center located $\sim 0.6 H$ over the ground and $\sim H$ apart from the building downstream. This recirculation vortex extends to a stagnation/convergence zone (the reattachment point) present in the center of the wake, $\sim 0.2 H$ thick, located $\sim 1.2 H$ downwind. Beyond this convergence zone, the wake assumes an almost constant height of $\sim 0.7 \mathrm{H}$ and extends to the outflow model boundary.

A comparison of Figs. 4 and 5 reveals differences in flow structures under neutral $(S=0)$ and moderately stable equilibrium $\left(S=3 \times 10^{-5} \mathrm{~m}^{-1}\right)$, as seen in vorticity field. Along vertical building leading edges, a separation of the boundary layer with a reverse flow occurs at $100 \mathrm{~m}$ in the neutral case and at $150 \mathrm{~m}$ in the moderately stable one. In the neutral case, the recirculation zone is also located higher (vortex center at $150 \mathrm{~m}$ ) than in the moderately stable case (vortex center at $100 \mathrm{~m}$ ). Under stable conditions, the downstream recirculation zone is tilted downward. Vertical cross-sections in Figs. 4 and 5 show that the vorticity wake within the $100-150 \mathrm{~m}$ height range propagates nearly horizontally in the neutral case while it becomes undulated when stability effects appear.

In cross-sections perpendicular to the undisturbed flow direction $(Y Z)$, differences in flow structures are even more pronounced (Figs. 6, 7). The neutral flow just behind the building is dominated by YZ-plane divergence; rotational components are present in the building wake and inside barely discernible horseshoe vortices that surround the lower and the main part of the building (Fig. 6, upper right panel). The arms of the horseshoe vortices converge to the stagnation zone and quickly dissipate in the turbulent wake. Beyond the convergence zone, the vorticity pattern becomes irregular and the flow exhibits convergence in $Y Z$ cross-sections. In contrast, in the moderately stable case the horseshoe vortex 

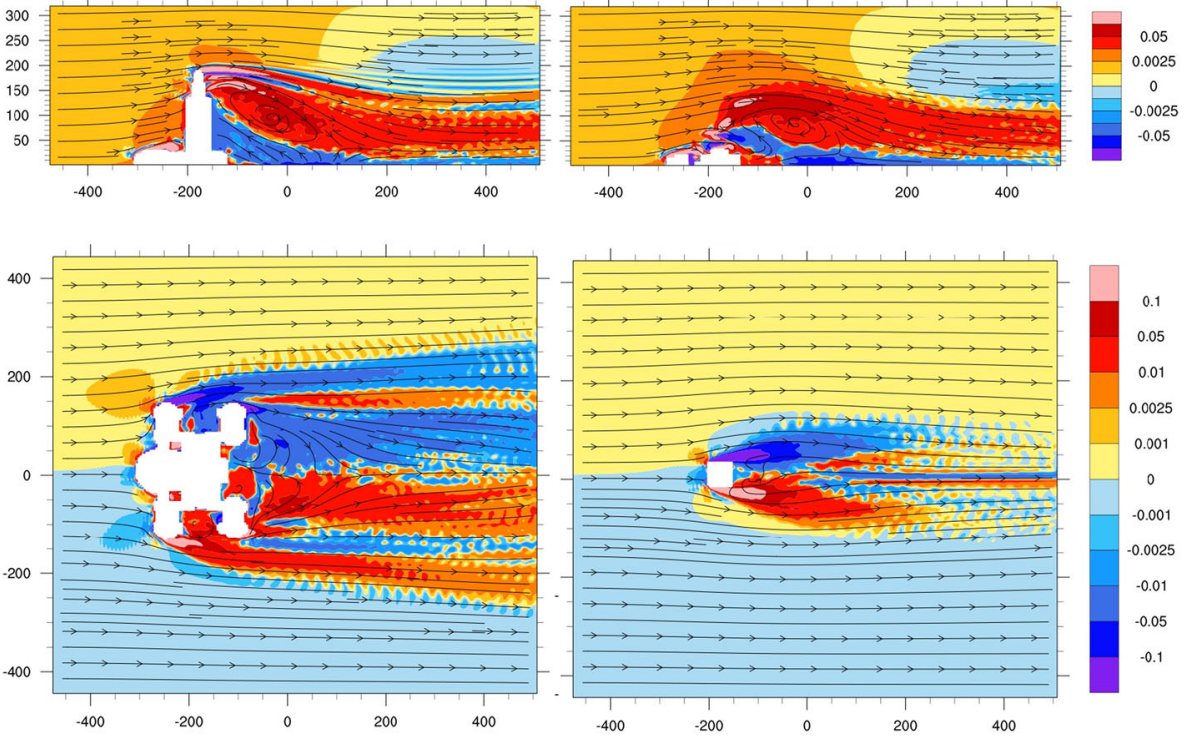

Fig. 5 As Fig. 4 but for the moderately stable case $S=3 \times 10^{-5} \mathrm{~m}^{-1}$

is well developed and shapes the vorticity-dominated flow pattern as observed in $Y Z$ crosssections. Just behind the building, the $Y Z$ cross-section exhibits two well-defined coherent circulation patterns which constitute arms of the horseshoe vortex, with their centers located initially at a height of $\sim 50 \mathrm{~m}$. These arms extend downwind, slightly slantwise downward and outward from the wake centerline. Interestingly, a new vortex system
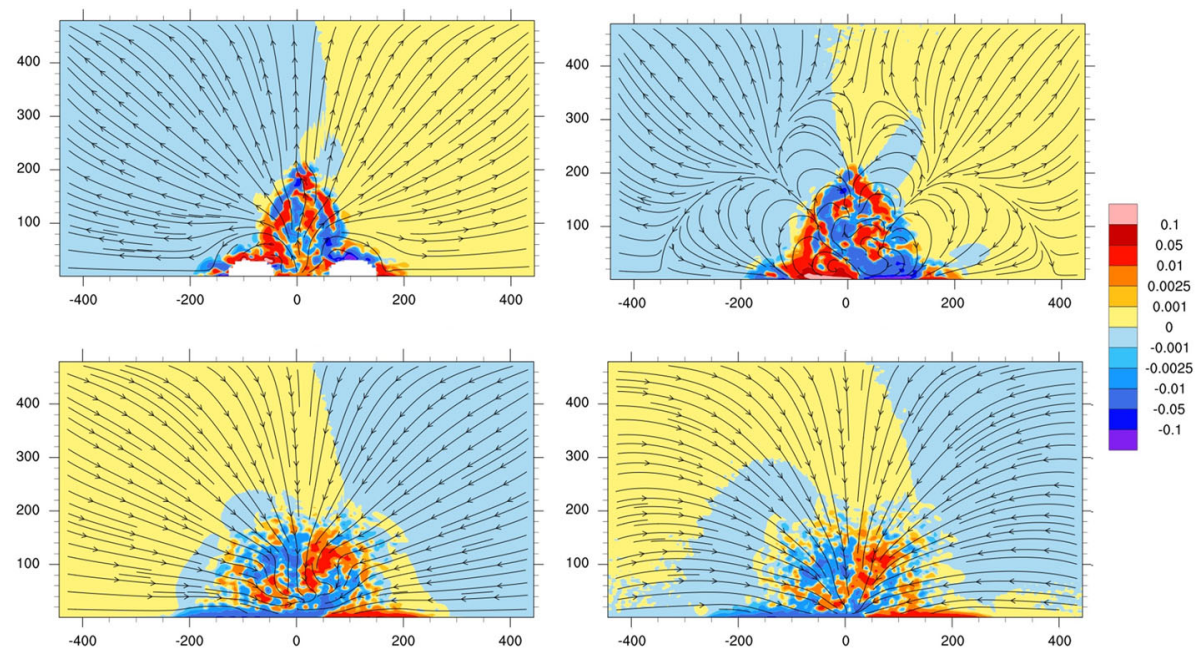

Fig. 6 Neutral case-lateral cross-section of the vorticity field at four locations behind the building: at $x=-110 \mathrm{~m}$ (near the building wall), $-30 \mathrm{~m}$ (center of the recirculation zone), $360 \mathrm{~m}$ (wake center), and $506 \mathrm{~m}$ (outflow boundary) 

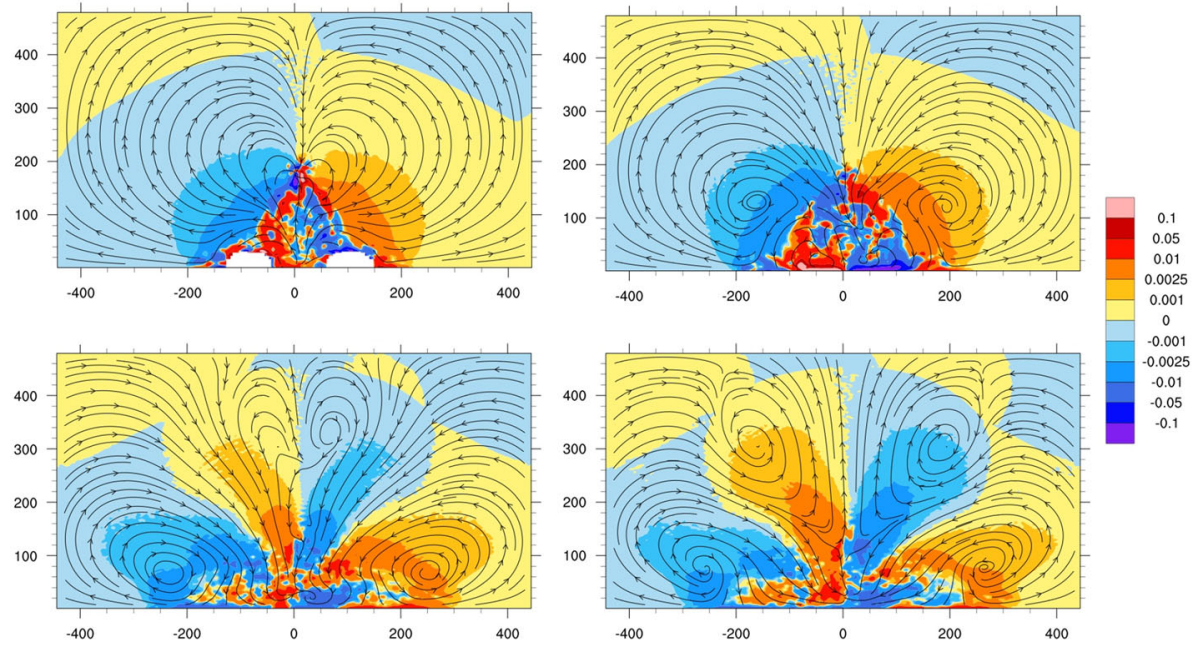

Fig. 7 As Fig. 6, for the moderately stable case $\left(S=3 \times 10^{-5} \mathrm{~m}^{-1}\right)$

develops above the stagnation region and extends downwind, forming a V-shaped pattern in horizontal planes. Further downwind, the two pairs of vortices form a characteristic pattern in $Y Z$ cross-sections, resembling a butterfly's wings (Fig. 7, lower right panel).

\section{Mean flow structures at moderate and low Froude numbers}

To examine the influence of atmospheric stability on the development of salient structures of the mean flow at moderate to low Froude numbers $(\mathrm{Fr}<1)$, we found it necessary to expand the computational domain downwind, doubling the number of grid steps in the $X$ direction to the total of 512 points (corresponding to $2044 \mathrm{~m}$, roughly ten times the building height). Figure 8 displays patterns of vertical vorticity component at a fixed height of $100 \mathrm{~m}$, for different stability conditions. As can be seen, the width of the wake at this height-roughly $400 \mathrm{~m}$ - does not significantly depend on stratification, although some changes of the shape of the wake and differences in the layout of vorticity filaments may be noticed. Maps plotted at lower levels display similar wake extents.

Figures 9 and 10 present a comparison of vertical velocity and potential temperature departure in the vertical center plane of the building wake. Leeward of the building, two dominating motion structures are evident: a recirculating vortex in the close vicinity, and a waveform flow extending further downstream. Areas of rising and sinking motions are interspersed with bands of positive and negative potential temperature departure, signaling the ongoing conversion between the kinetic and potential energy. This waveform motion is apparently excited by the forced ascending motion windward of the building and the compensating sinking at the lee side. Clearly, it can be identified as a stationary gravitational lee wave, extensively discussed in mountain meteorology (e.g. [61, 62]).

While the wave appears to be attached to the obstacle - in other words, stationary in an Eulerian reference frame-it actually may be considered as propagating upstream with its phase speed equal to the wind speed ([48, p. 48]). For an internal purely gravitational plane wave, the linear theory yields the dispersion relation (e.g. [48]) 

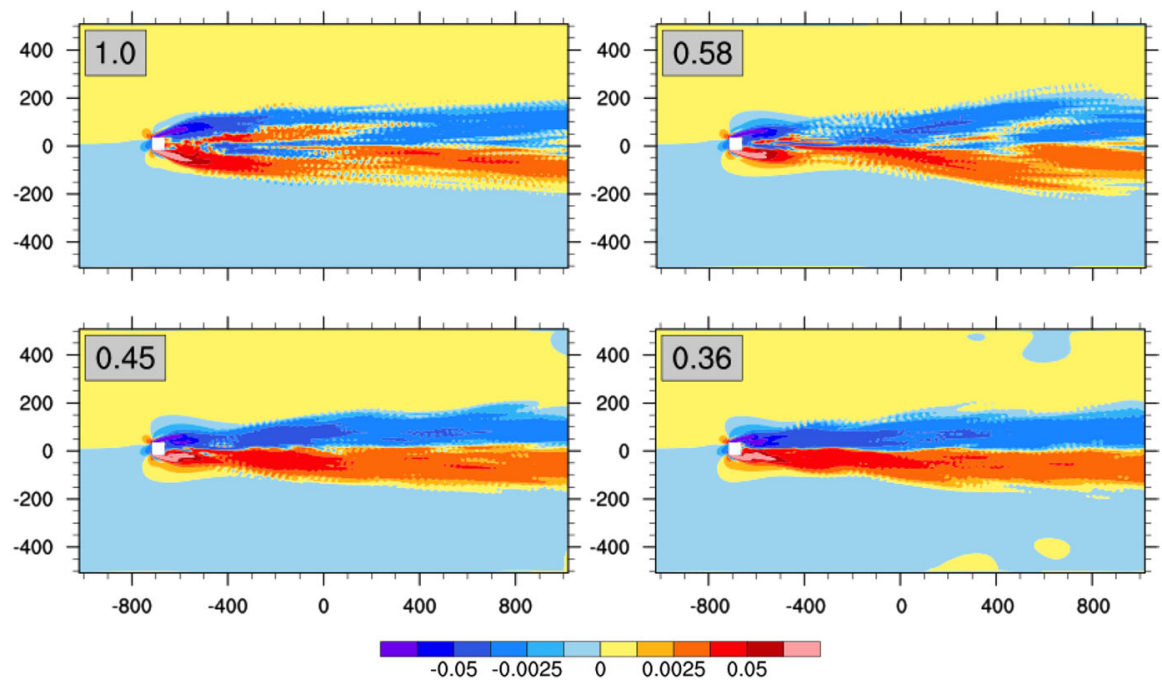

Fig. 8 Vertical vorticity component at $100 \mathrm{~m}$ height in different stability conditions (values of Froude number are shown in upper left corners of each panel)
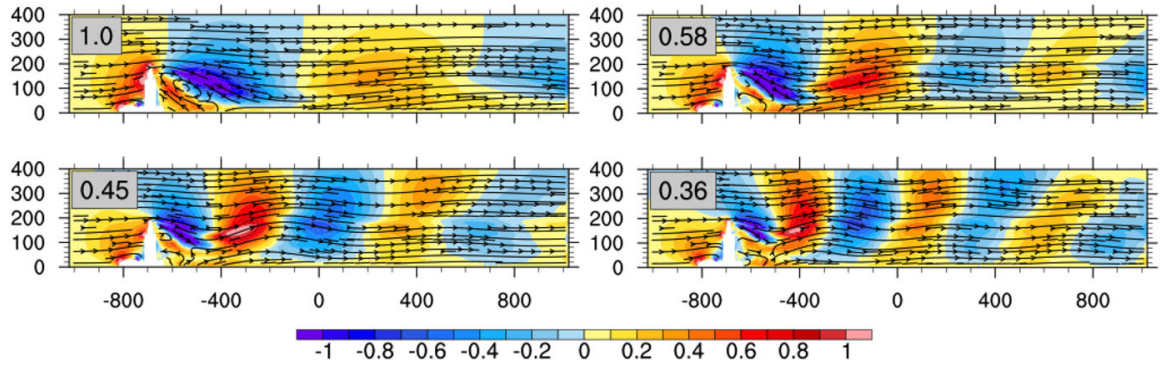

Fig. 9 Vertical velocity $\left(\mathrm{m} \mathrm{s}^{-1}\right)$ and streamlines in a vertical cross-section running along the centerline of the wake. Values of the Froude number are shown in upper left corners of each panel
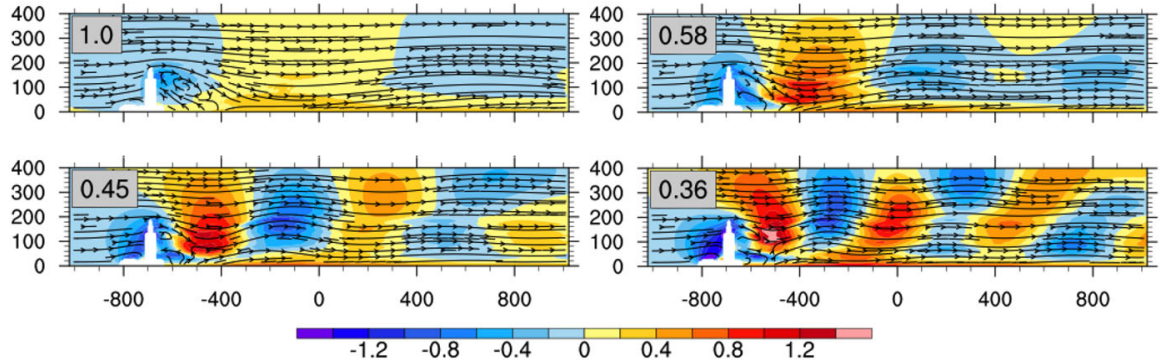

Fig. 10 Potential temperature departure $(\mathrm{K})$ and streamlines in a vertical cross-section running along the centerline of the wake. Values of the Froude number are shown in upper left corners of each panel 


$$
\frac{\omega}{k}= \pm \frac{N_{B V}}{\sqrt{k^{2}+m^{2}}}
$$

in a Cartesian coordinate frame with its horizontal $X$-axis located in the vertical plane containing the direction of wave propagation. Here, $\omega$ is the wave frequency, and $k$ and $m$ are the horizontal and vertical wavenumbers, correspondingly.

Recalling that in our case the phase speed $\omega / k$ is equal to the wind speed, one might obtain the length

$$
\lambda_{x}=\frac{2 \pi u}{N_{B V}}
$$

of a horizontally propagating wave. Table 1 displays numerical values of $\lambda_{x}$ calculated from this equation using the basic undisturbed flow velocity $u=3 \mathrm{~m} \mathrm{~s}^{-1}$. These values almost perfectly match wave lengths that can be read from Figs. 9 and 10 .

Our results can also be compared to previously published works on low Froude number flows around isolated mountains, in particular to the linear solution presented by Smith [64] and to the numerical simulation by Smolarkiewicz and Rotunno [70]. To facilitate the comparison, isentropic surface heights were calculated and plotted (Fig. 11). In our experiments, the ambient potential temperature was set to decrease linearly with height, starting from a common fixed value at $z=0$. Hence, there is no common value of $\theta_{e}$ that could be used for plotting isentropic surfaces located at similar heights. Rather than, $\theta_{e}\left(z=z_{\text {ref }}\right)$ for a specified reference height $z_{\text {ref }}$ was used for each individual plot.
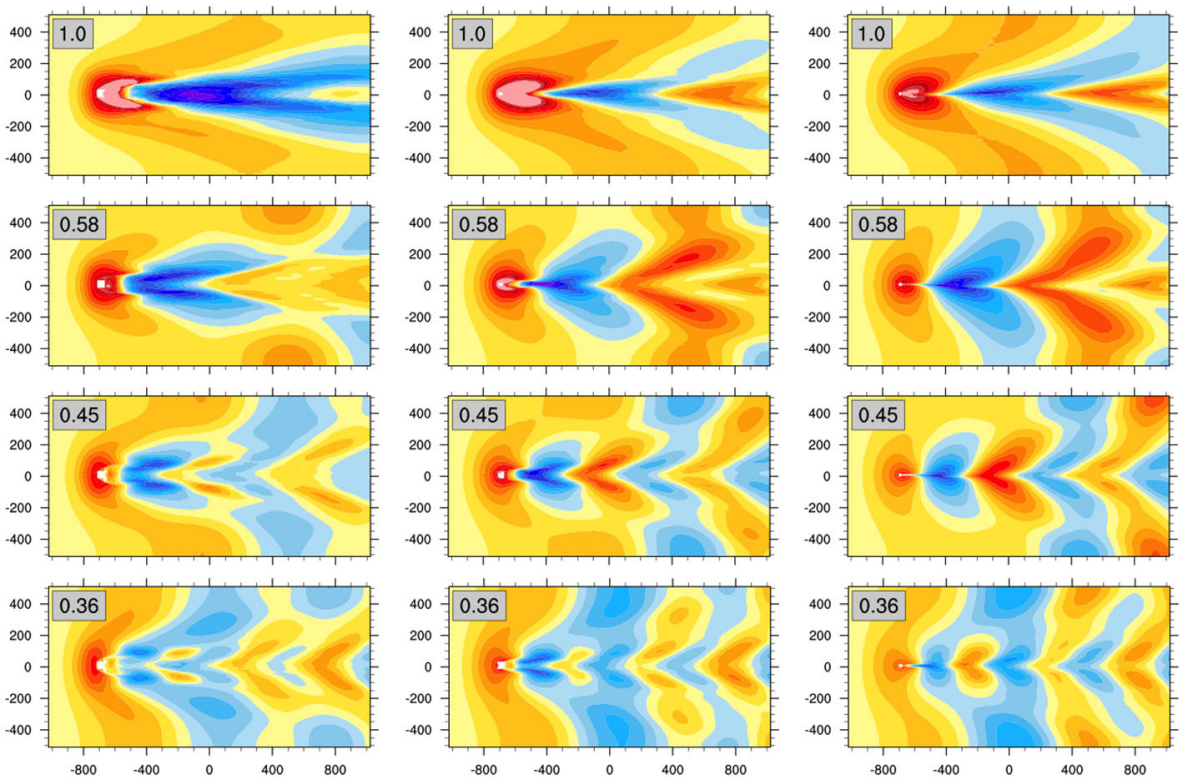

$\begin{array}{lllllllllll}20 & 28 & 36 & 44 & 52 & 60 & 68 & 76 & 84 & 92 & 100\end{array}$

$\begin{array}{lllllll}60 & 68 & 76 & 84 & 92 & 100108116124132140\end{array}$

100108116124132140148156164172180

Fig. 11 Elevation of isentropic surfaces in mean flow where $\theta=\theta_{e}(z=60 \mathrm{~m})$ (left column), $100 \mathrm{~m}$ (center column) and $140 \mathrm{~m}$ (right column) for different stability conditions as reflected by the Froude number (values shown in upper left corners of each panel) 
Apparently, patterns in Fig. 11 are superpositions of (at least) two major components-a much narrower, elongated wake shaped by the recirculation vortex, and a wider, more regular V-shaped pattern of gravity waves extending to the wake boundary. This pattern changes, however, outside the wake where wavefronts tend to assume direction perpendicular to the flow. This is particularly apparent in lower Froude number flows. One might note that this isentropic surface cuts through the main tall part of the building and also passes through the recirculation vortex. Above this part of the building, on the isentropic surface drawn for a larger reference height $\left(z_{\text {ref }}=140 \mathrm{~m}\right.$, rhs panels on Fig. 11) wave patterns become simpler and more regular. Conversely, patterns corresponding to $z_{\text {ref }}=$ $60 \mathrm{~m}$ (left panels) appear as more complex.

The linear theory by Smith [64] predicts the presence of an U-shaped depression in the lee of an isolated obstacle, with its arms running at a larger angle for greater heights ([64], Fig. 1a-c). Some of the forms presented here resemble these results, although a greater variability in wavefront shapes can be noticed, dependent on the value of the Froude number. A similar tendency of the angle between wavefront arms is apparent from comparison of $z_{\text {ref }}=100 \mathrm{~m}$ to $z_{\text {ref }}=60 \mathrm{~m}$ results in Fig. 11 .

Smolarkiewicz and Rotunno ([70], their Fig. 2) presented patterns of vertical displacement of an isentropic surface at various reference heights, for different values of the Froude number. Again, while exact comparison is impossible because of the different approach to selecting reference heights and the scaling adopted for presentation, we should note similarities in patterns of depressions and crests. Also in agreement with their principal results (their Fig. 4), we observe stronger development of the pair of vertical vortices in the building wake, some $50-100 \mathrm{~m}$ behind the building - at lower Froude numbers, beginning with $\mathrm{Fr}=0.58$. Finally, vertical cross-section in the wake centerplane (their Figs. 3c, 4) reveal the same features as presented in our Figs. 9 and 10.

While the Froude number given by Eq. 13 is primarily defined as a ratio of inertial and buoyancy forces, an interesting interpretation in terms of mechanical energy is also possible. By letting the disturbance of a potential temperature of a vertically displaced parcel to be equal to

$$
\theta^{\prime}(z)=-z \frac{\partial \overline{\theta_{e}}}{\partial z}
$$

and recognizing further that the work done by moving the parcel against the buoyancy force is

$$
d L=-\frac{g}{\theta} \theta^{\prime} \delta z
$$

which, upon substituting the ambient temperature profile from (16) with a fixed potential temperature gradient, and integration over a height range from 0 to $H$ yields

$$
\Delta L=-\frac{g}{\theta} \frac{\partial \overline{\theta_{e}}}{\partial z} \frac{H^{2}}{2}=\frac{N_{B V}^{2} H^{2}}{2} .
$$

Comparing Eq. 13, we see that the square of the Froude number can be seen as a ratio of the kinetic energy of a parcel moving with the mean flow, to the potential energy gained by a forced displacement of the parcel during its rise from the obstacle base to its top.

At the same time, the square of the numerator represents the (doubled) kinetic energy of the parcels engaged in the mean flow, per unit mass. Hence, $\mathrm{Fr}=1$ corresponds to a height of a hypothetical obstacle that can be entirely overflown by air stream owing a given initial 
velocity, in a stably stratified environment characterized by a certain value of the BruntVäisälä frequency. In other words, $\mathrm{Fr}=1$ marks the maximal possible vertical displacement of an ascending fluid particle in a steady-state adiabatic flow of ideal gas. For a fixed obstacle height, there is a qualitative difference between flows at $\mathrm{Fr}>1$ and at $\mathrm{Fr}<1$ : in the former situation, a vertical movement of a parcel descending from a top of the obstacle is limited by the presence of ground surface, whereas in the latter one it becomes limited by buoyancy forces and cannot reach the ground. For this reason, we observe the vertical extent of the lee recirculation vortex decreasing with $\mathrm{Fr}$ - in contrast to high Froude number flows discussed in the previous section. Also, we see that the wave height (defined as a difference in height of crests and troughs of the undulated isentropic surface) decreases with Fr.

An interesting question regards the possible interactions between vortex structures and lee waves. For a better insight, vertical velocities were mapped at an isentropic surface computed for $z_{\text {ref }}=100 \mathrm{~m}$ (Fig. 12). Although the isentropic surface is undulated, the differences between vertical velocities mapped at this surface and at a fixed height of 100 $\mathrm{m}$ (not shown) are barely noticeable, except for the immediate vicinity (a few gridpoints) of the building. Not surprisingly, vertical velocity patterns are closely related to forms visible in the isentropic surface elevation map (Fig. 11); as the motion has been modelled as nearly adiabatic, vertical velocities are nearly proportional to the local inclination of the height of the isentropic surface. On the other hand, spatial changes in vertical velocity may signal the presence of eddies rotating in vertical plane. To isolate the rotational motion, longitudal $(X)$ vorticity component was calculated at a fixed height $(100 \mathrm{~m})$ and presented in Fig. 13. This set of plots is supplemented by a set of cross-flow (YZ) sections (Fig. 14) drawn at $x=576 \mathrm{~m}$ (approx. $1.2 \mathrm{~km}$ behind the building).

As can be seen, vorticity patterns in the far-field are symmetric, but highly complex. Essentially, every crest of the lee wave inside the wake is associated with a V-shaped pair of vortices with their axes slightly inclined with respect to the terrain. At a moderate
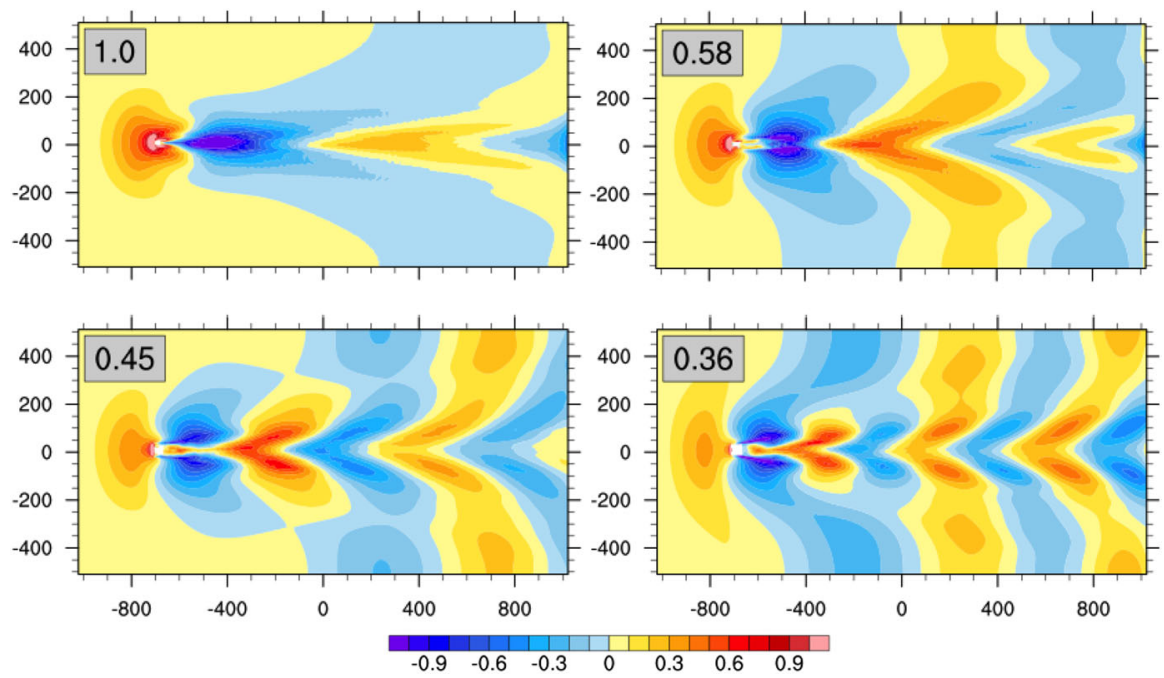

Fig. 12 Vertical velocities $\left(\mathrm{m} \mathrm{s}^{-1}\right)$ at the isentropic surface $\theta=\theta_{e}(z=100 \mathrm{~m})$. Froude number values are shown in upper left corners of individual panels 

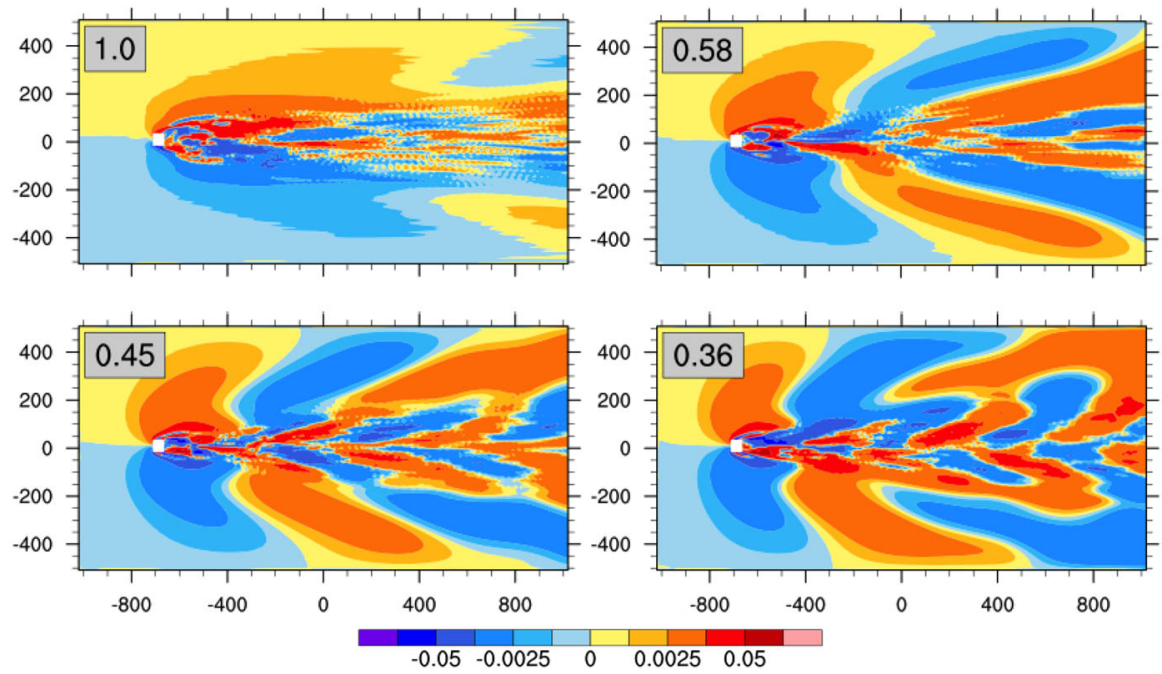

Fig. 13 Streamwise $(X)$ vorticity component $\left[\mathrm{s}^{-1}\right]$ at $100 \mathrm{~m}$ level. Froude number values are shown in upper left corners of individual panels
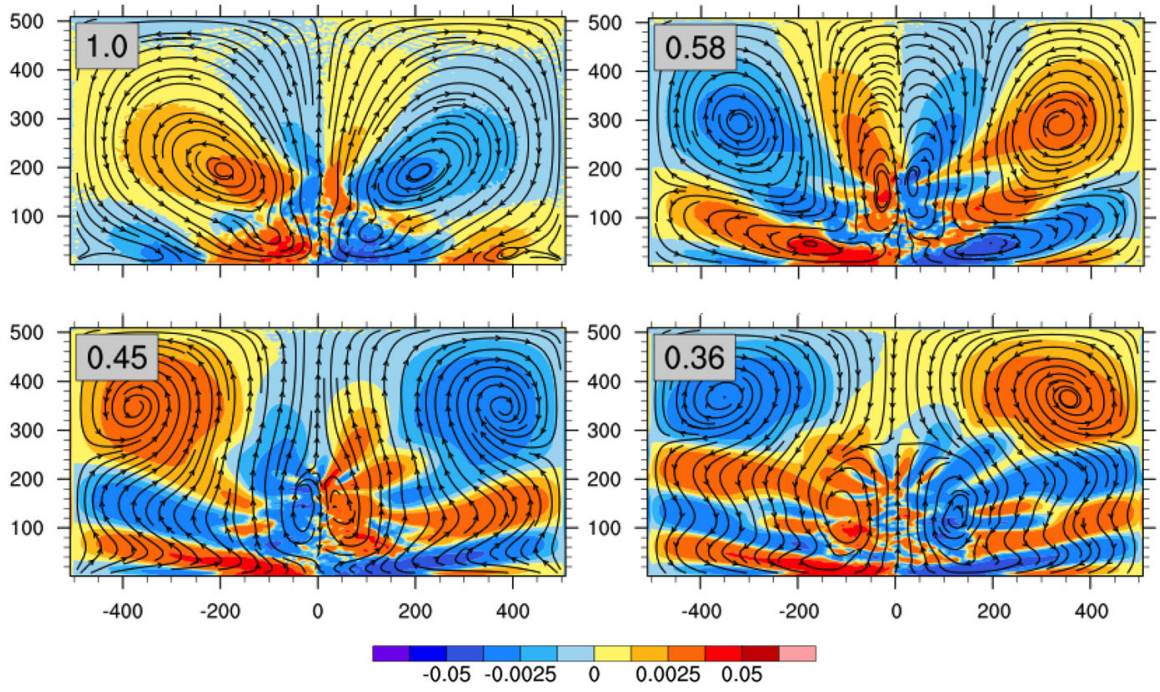

Fig. 14 Streamwise $(X)$ vorticity component $\left[\mathrm{s}^{-1}\right]$ at a cross-flow section located at $x=576 \mathrm{~m}$. Froude number values are shown in upper left corners of individual panels

stability, the leading U-shaped horseshoe vortex extends to a few building heights downwind, but, as the lee waves became shorter with decreasing Fr, multiple vortex pairs intertwined with waves appear. The cross-flow section drawn at approximately $1.2 \mathrm{~km}$ behind the obstacle shows several pairs of vortices, and the overall complexity increases with stability. 


\section{A comparison with flow around a cuboidal building}

A vast majority of studies on flows around buildings focused on simple geometries. A simulation of strongly stratified flow around a cuboidal building of a size comparable to PKiN's has been done to complement the results presented in the former sections and to facilitate comparisons with the literature data. Dimensions of a cuboid, $40 \times 44 \times 132 \mathrm{~m}$ were chosen to match the central, tall part of the PKiN building-without its broad base, pavilions, the turret and the spire. In this simulation, external flow conditions were kept identical to the formerly discussed setups, and the stratification parameter matched case \#13 from Table 1, that is, $S=2 \times 10^{-4} \mathrm{~m}^{-1}$.

The temporally-averaged elevation of an isentropic surface $\theta=\theta_{e}(z=100 \mathrm{~m})$ is shown in Fig. 15 (left panel). These results can be compared to the corresponding element in Fig. 11 (middle column, last row). In the nearest vicinity of the building, some 50 metres apart, the wake is more elongated in the direction of flow while in the case of a real building shape the spanwise distortion is remarkably more pronounced. Further downstream, a V-shaped wave pattern similar to the one previously described can be seen, however the spanwise propagation of disturbances is narrower. Except for these details, the wave patterns and wave lengths in both cases are quite similar.

Averaged vertical velocities mapped on this isentropic surface (Fig. 15, right panel; compare to Fig. 12, bottom row, right column) show more pronounced differences. In case of a narrow cuboid building, vertical velocities are generally lower than $\pm 0.1 \mathrm{~m} \mathrm{~s}^{-1}$ outside a roughly $300-\mathrm{m}$ wide wake. The real building with its wide base and pavilions excites vertical motions with velocities ranging to and over $\pm 0.3 \mathrm{~m} \mathrm{~s}^{-1}$, at a distance of $300-400 \mathrm{~m}$ from the wake center. In the nearest vicinity of the building, there are also substantial differences of vertical velocity along the centerline of the wake, which can be explained by the impact of the turret.

The vertical cross-section drawn along the wake centerline (Fig. 16; compare to Fig. 9, bottom row, right column) reveals an area of stronger ascending flow on the windward side of the cuboidal building near the roof level. Also, the recirculation vortex on the lee side is more pronounced and has just one, not two bands of maximal descending velocities. The position of the convergence point discussed in Sect. 3 remains unchanged, and the wave pattern remains nearly identical. Also, vorticity patterns (Fig. 17) beyond the immediate
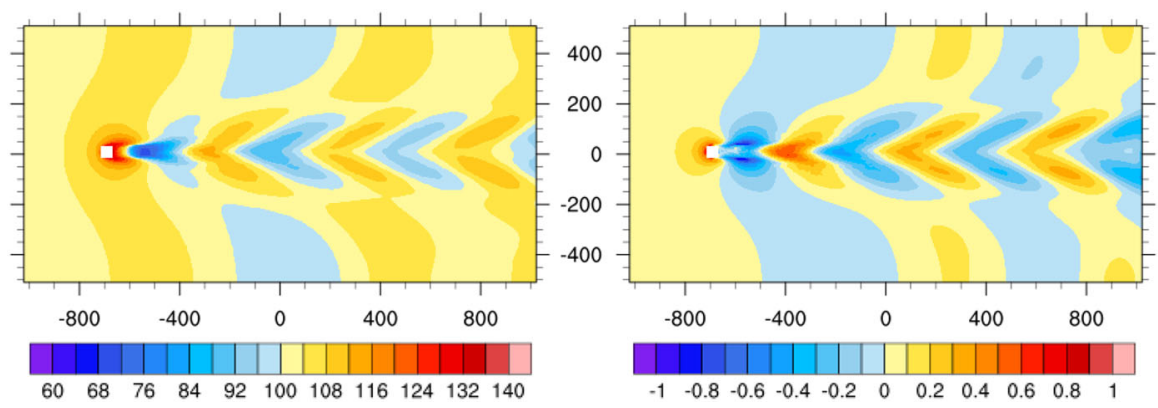

Fig. 15 Elevation of isentropic surface $\theta=\theta_{e}(z=100 \mathrm{~m})$ in mean flow around a cuboidal building for $S=2 \times 10^{-4} \mathrm{~m}^{-1}$ (left panel); and vertical velocities mapped on the isentropic surface $\theta=\theta_{e}(z=100 \mathrm{~m})$ (right panel) 


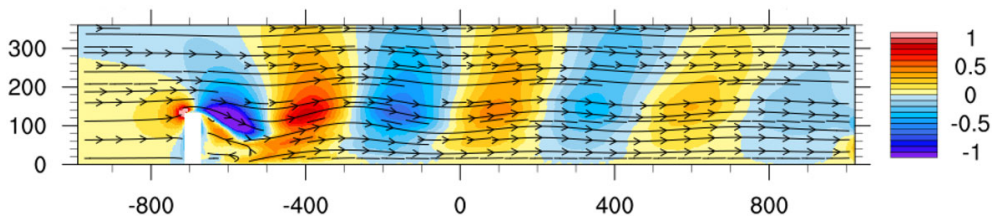

Fig. 16 Vertical velocity $\left(\mathrm{m} \mathrm{s}^{-1}\right)$ and streamlines in the flow around a cuboidal building, in a vertical crosssection running along the centerline of the wake

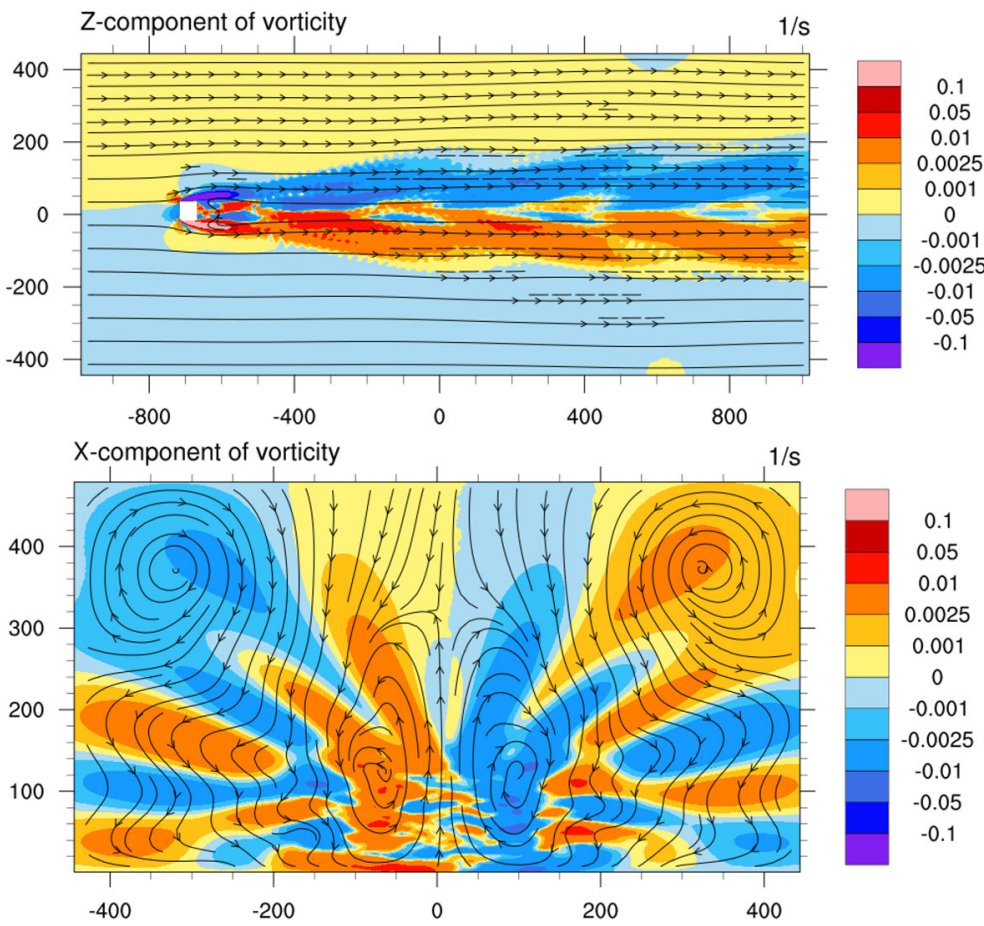

Fig. 17 Vertical vorticity component at $100 \mathrm{~m}$ height (upper panel) and streamwise $(X)$ vorticity component $\left(\mathrm{s}^{-1}\right)$ at a cross-flow section located at $x=578 \mathrm{~m}$ (lower panel), in the flow around a cuboidal building

vicinity of the building remain substantially similar. In the simpler cuboid building case, the vertical vorticity component exhibits a regular pattern, apparently associated with the wave pattern.

\section{A case study: January 1, 2016}

To recognize flow structures in a more realistic situation, a case of strong surface-based inversion that evolved over Warsaw during the night of December 31, 2015 to January 1, 2016 was chosen as the background for numerical simulation. According to the 00 UTC 
sounding at the aerological station at Legionowo (WMO code 12374), located approximately 20 kilometers north of the PKiN building, the inversion extended to $320 \mathrm{~m}$ above the ground, with the temperature increasing from $-10.1^{\circ} \mathrm{C}$ at the screen level to $-3.5^{\circ} \mathrm{C}$ at the inversion top. This corresponds to a $9.8 \mathrm{~K}$ increase of the potential temperature $\left(S=1.15 \times 10^{-4} \mathrm{~s}^{-1}\right)$. The wind speed was varying from 2 to $8.5 \mathrm{~m} \mathrm{~s}^{-1}$ between these two levels, veering by $60^{\circ}$ within the first 160 metres, then backing by $10^{\circ}$ to the inversion top. Thus, the value of the Froude number that could be assigned to these conditions is close to one. Above the inversion, the potential temperature profile exhibited a weak growth with height within the entire range of heights encompassed by the model domain.

A proper representation of ambient meteorological conditions and boundary conditions in a building-scale model is not a straightforward task. While nesting within a mesoscale meteorological model such as in [9] appears to address most of the issues, its high demand for resources and data precludes its broader application. Moreover, in reality we deal with a multiscale problem including inter-scale feedbacks, hence a simple one-way nesting still retains idealization and limitations. When effects of isolated buildings are investigated, it seems more appropriate to assume homogeneouos meteorological conditions on the scale of the entire model domain. The effect of homogeneity of inflow conditions and their consistency with near-wall parametrizations was recently discussed by $\mathrm{Ai}$ and Mak [1] in the simulations of flow around a cuboid, done using a variable-mesh RANS $k-\varepsilon$ model. It was found that (i) boundary-layer inhomogeneities had significant impact on the flow, (ii) the near-wall treatment had a considerable effect on the reattachment length on the roof, but small on the reattachment length in the wake, and (iii) in general, the RANS model poorly predicted the size of recirculation regions on both the roof and the wake. With respect to these results, our approach differs in several aspects, as we are primarily interested in investigating flow features developing at larger distance from the building, and our focus is on stably stratified flow where the frictional effects on horizontal surfaces are much smaller than in neutrally stratified flows. In our LES approach, resolved-scale turbulent motions excited by shear instability are explicitly calculated, while the subgrid-scale turbulence parameterization has lesser importance (see e.g. [2]), especially under stable conditions. Further, the IMB method used in our study accounts for flow impingement effects by introducing reaction forces into momentum budget equations, not by directly imposing boundary conditions reflecting wall impermeability and surface friction. In this aspect our results are dependent (in a quantitative way) on the grid resolution near the building wall, however the decomposition of flow variables into the environmental/ambient state and its perturbations provides a convenient way of setting horizontally homogeneous forcing within the entire model domain.

With the above factors in mind, we have opted for a relatively simple modelling strategy. First, the potential temperature and wind profiles obtained from sounding are entered as initial conditions into a single-column, high-resolution turbulence-closure model. The purpose is twofold-to provide a self-consistent interpolation to the dense model grid, and to filter out irregularities originating from local flow perturbations or measurement errors. The single-column model used here solves momentum and heat transport equations in a vertical by an implicit, Crank-Nicolson type finite-difference method, using staggered grid with variable spacing that ranges from a few centimeters close to the ground to ten metres at the top (set at $1 \mathrm{~km}$ ). A log-linear transformation of height, originally proposed by Taylor and Delage [75] is applied to minimize truncation errors arising from nonlinear variability of meteorological elements close to the ground. 

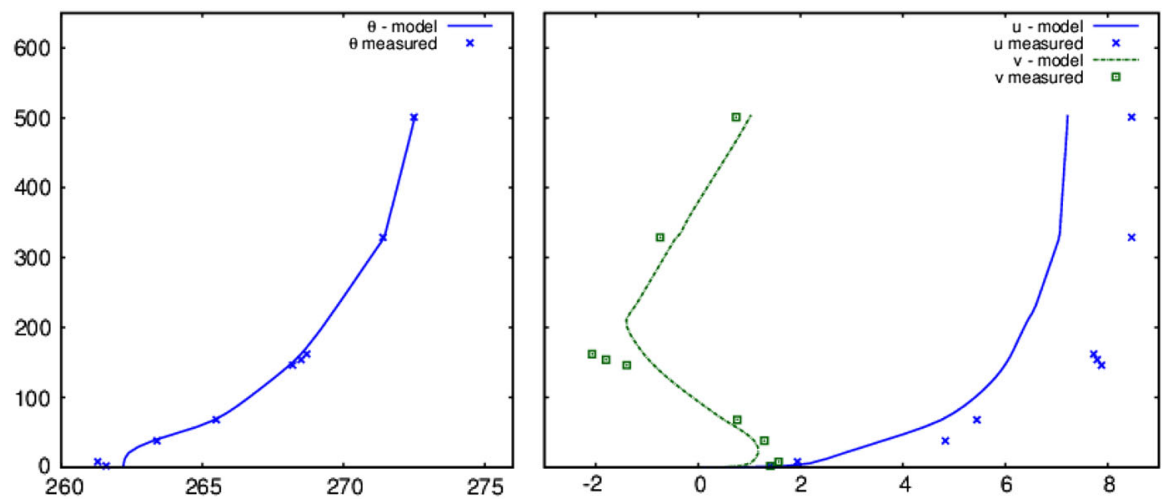

Fig. 18 Profiles of potential temperature (left panel) and wind components (right) as calculated by a singlecolumn, turbulence-closure model adopted as the background (environmental) conditions for the LES in this case study. Profiles are based on aerological sounding at Legionowo (WMO station code 12374), January 1, 2016, 00 UTC

The turbulent transport is modelled using the Level 3 second-order turbulence closure scheme, as introduced by Mellor and Yamada [41, 42], with realizability limitations and model constants developed by Nakanishi and Niino [47]. For more details, see [37]. While integrating this model with constant boundary conditions, we found it sufficient to run the model for just 5 mins with a one-second time step. Figure 18 displays the assimilated profiles along with the raw sounding data.

Next, the resulting potential temperature and wind velocity profiles are used as the "environmental" state variables $\left(\theta_{e}, \mathbf{v}_{e}\right)$ in the EULAG; while doing so, we adjust the mean flow direction to our domain (along the $X$-axis). As mentioned above, using "environmental" profiles provides horizontally homogeneous background flow that remains in a steady, quasi-equilibrium state; this also ensures the proper forcing for perturbations resulting either from interactions with an obstacle or from flow instability. Note, that while there are several methods for generating turbulent perturbations in the inflow and the simulated flow structure exhibits response to the choice of a particular method, the currently available intercomparisons [2,89] are limited and do not provide clear evidence of general superiority of any of the methods tested. Therefore, the smooth inflow option was used in all of our present computations. As with no-slip type boundary conditions, we entirely rely on the internal generation of turbulence due to the strong shear maintained by the background flow.

Further, an equilibrium state check in an empty computational domain is done, as recommended in [89] in order to ascertain that homogeneity conditions are actually maintained during the model integration. The spin-up period is determined by observing formation of quasi-stationary flow regime, and the model integration period is chosen to match the domain cross-over time.

Figure 19 shows streamlines and vertical velocities at several model levels. As can be seen, the flow is dominated by significant wind turning with height, and patterns of flow and vertical velocity do not match each other. At higher levels, traces of wave motion are discernible. This weak waveform motion is also visible in the vertical structure of flow and 

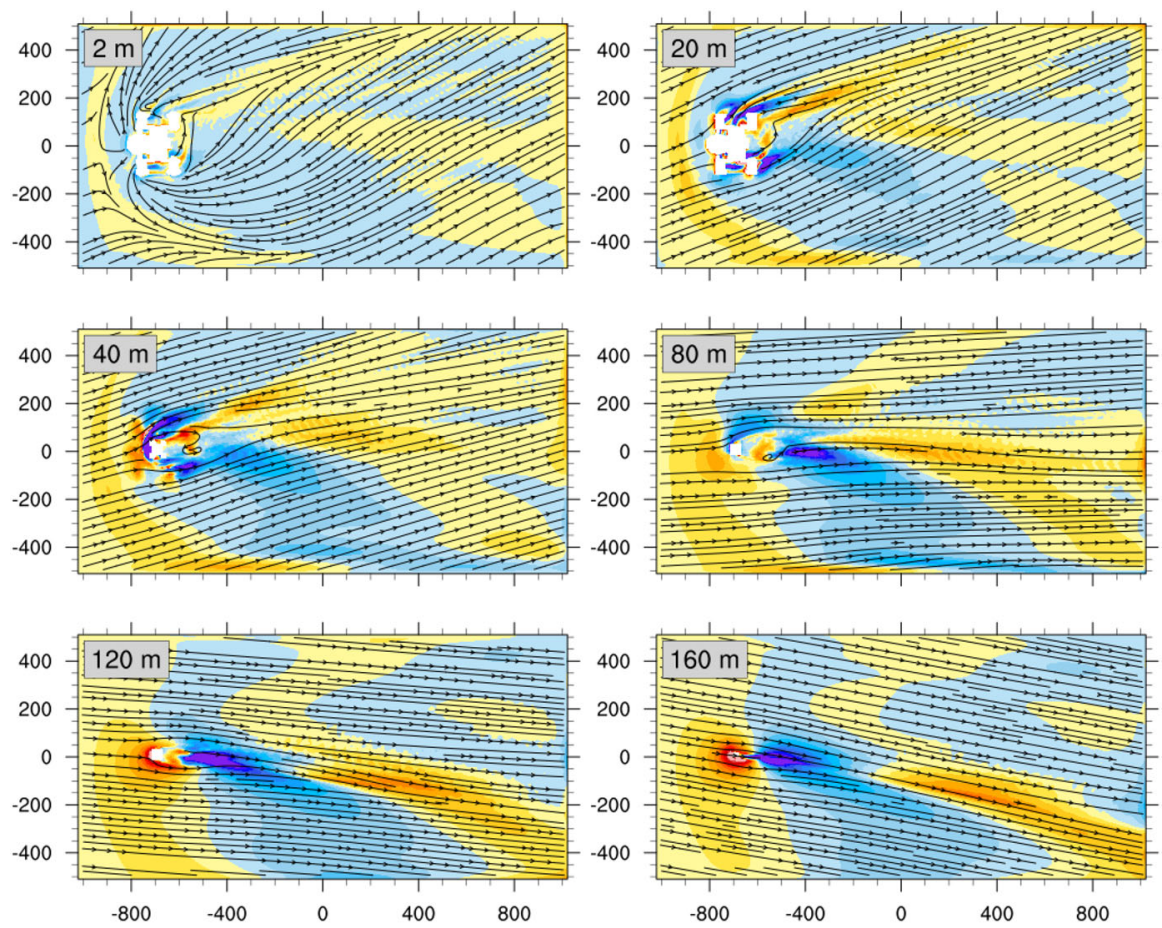

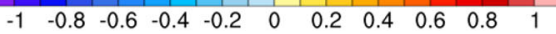

Fig. 19 Vertical vorticity component at different levels (as shown in panel insets) and $X Y$-streamlines

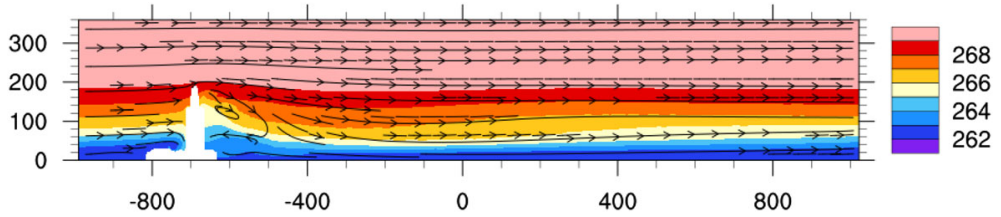

Fig. 20 Flow structure and the potential temperature in a $X Z$ cross-section running along the centerline

potential temperature (Fig. 20). Cross-sections perpendicular to the principal flow direction (YZ, Fig. 21) show that the helical structures of motion forming at both sides of the building and later on both sides of the wake-are actually driven by the change of wind direction with height. The building wake is remarkably skewed by the wind shear; at larger distances from the obstacle it turns into a centerline of a helical vortex extending along the mean wind direction at $100 \mathrm{~m}$ level. Overall, it appears that the vortical flow structures are formed mainly by the wind shear, and the obstacle triggers the separation of eddies. 

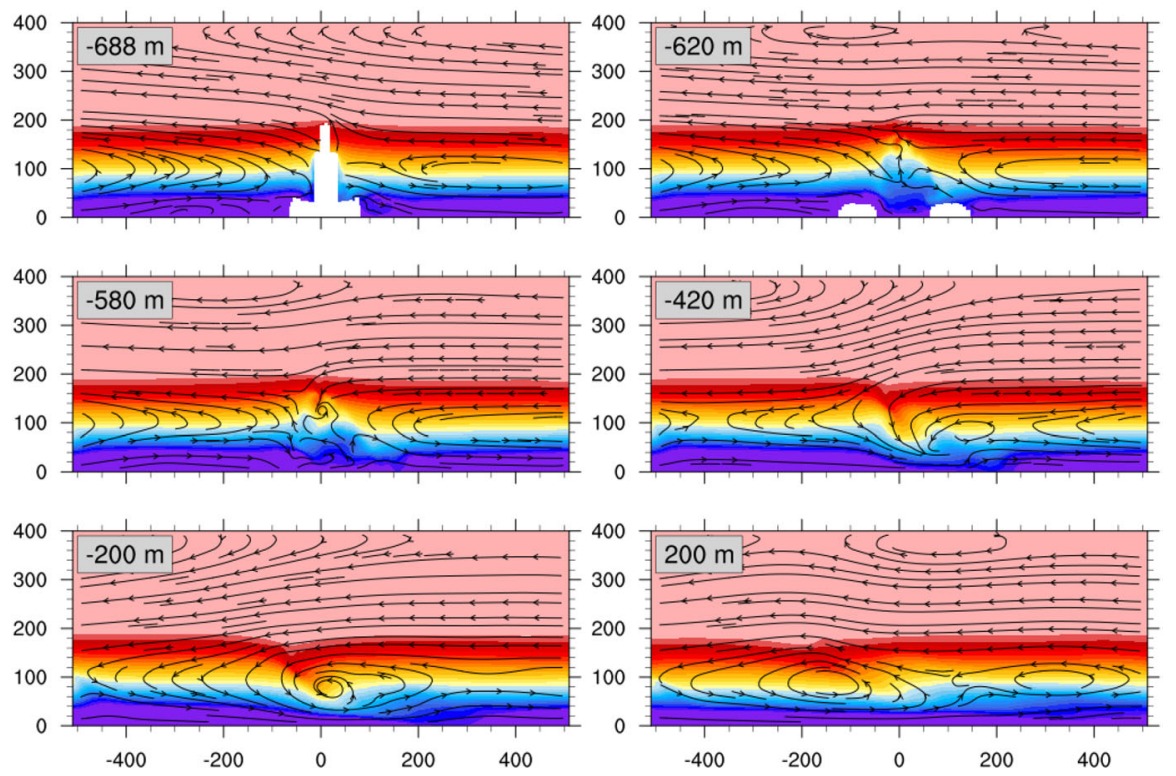

263

264

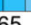

266

267

268

269

Fig. $21 Y Z$ cross-sections through the potential temperature field and streamlines at several distances from the domain center (shown in panel insets)

\section{Conclusions}

We have performed a series of idealized numerical experiments designed to explore effects of static stability of the atmosphere on flow structures arising after passing around an isolated, tall building. Many of the flow features identified in the results, such as the horseshoe vortex, the lee recirculation vortex, lee waves and vortex pairs have been previously known from wind tunnel and laboratory experiments, numerical simulations and linear theories. The response to stability changes observed in our results qualitatively agrees with similar theoretical and computational studies on mountain flows. In particular, we observe weakening of trailing vortices with decreasing stability, in agreement with windtunnel studies by Kothari et al. [30]. We have also found a good quantitative agreement between computational results and linear gravity wave theory in predicting the wavelength of internal gravity waves identified as isentropic surface disturbances in the wake.

The effects of stratification on the vertical distribution of drag force and turbulence around the building and their effect on dispersion of pollutants require further attention. Apparently, flow structures at low Froude numbers are more complex, while the absolute values of vorticity do not decrease remarkably. Therefore, turbulence generation associated with local shear and gravity wave breaking should continue to occur in the wake, even at relatively large distances from the building.

An interesting aspect of the presence of standing gravity wave in the wake is its interaction with vortex structures. Our results reveal a close link between V-shaped waveforms filling the wake and vortices spreading out from the centerline of the wake intertwined with waves. With the Froude number decreasing, gravity waves become 
shorter and more vortices appear. The resulting vortex pattern becomes highly complicated, yet still regularly organized. Wave motion becomes apparent when the Froude number turns sufficiently small (say, for $\mathrm{Fr}<1$ ), a value somewhat smaller than reported in former studies [90] but qualitatively consistent. For $\mathrm{Fr}>1$, there is no pronounced relation between the extent of the lee recirculation vortex, the distance of the convergence zone from the building and stability. However, as the Froude number drops below unity, this situation changes. The vertical extent of the lee recirculation vortex decreases with the Froude number, as does the height of gravity waves. Further, a pair of symmetric vortices rotating in a horizontal plane appears and intensifies with increasing stability.

It has been also found that only major elements of the building structure noticeably affect the flow patterns at distances larger than the building height. Likely, a crude representation of the building shape may be sufficient for computations of the basic flow, except for the nearest vicinity of the building. In contrast, more realistic representation of ambient meteorological conditions - in particular, wind turning with height-may have tremendous impact on formation of flow structures behind the obstacle. As our results regard just a single case, this issue definitely deserves more research before attempting to generalize such a conclusion. Our research on this issue will be continued.

In this study, smooth/laminar inflow conditions were specified without explicit definition of the inflow turbulent intensity. The major disturbance and the source of instability is then the interaction of flow with the building structure. The inflow turbulence is more significant under neutral and unstable conditions when the amplitude of small disturbances can be preserved or amplified, respectively. Under stable conditions, buoyancy effects suppress small scale vertical motion, except for the areas where the turbulence is generated, as e.g., in surface shear layers. In pollutant dispersion problems, when a proper level of turbulence intensity is important at the upwind side of the building, the commonly used techniques are based on setting up simplified forms of inlet TKE (e.g. constant profile, [59]), dynamical recycling [78] or smooth inflow with generic downwind roughness elements [79]. Such conditions mostly affect the intensity of vertical mixing and the rate of boundary layer growth - factors determining concentrations of pollutants emitted within the urban canopy. These effects fall beyond the scope of this paper and will be investigated in subsequent studies.

While the good agreement between our computations and results of the linear theory [64] may seem tempting for practical applications of the latter, there are several caveats. First, the magnitude of the observed oscillations is not relatively small (for example, vertical velocities that exceed $1 \mathrm{~m} \mathrm{~s}^{-1}$ compared to the base flow velocity), hence the basic scaling assumptions of the linear theory are not generally fulfilled. Second, the presence of non-linear phenomena such as the recirculation vortex may affect the far-field solution. Third, as gravity waves are dispersive, their length should vary with the advective flow velocity varying gradually in the wake. While these effects may be quantified and justifiably neglected in certain situations, a more general practical applicability remains questionable. This calls for extending the current numerical analysis to a broader range of problems in urban fluid dynamics under stable thermal stratification.

Acknowledgments Large-eddy simulations that provided results presented here were prepared and carried out at the National Centre for Nuclear Research by Michał Korycki, as a part of his doctoral dissertation at the Warsaw University of Techology. The work was supported by the EU and MSHE grant $\mathrm{nr}$ POIG.02.03.00-00-013/09. The work of other authors was sponsored by their institutions from their statutory funds. The digital terrain maps used in this study have been provided by the City Hall of Warsaw. The authors are grateful to the anonymous reviewer whose comments have led us to extend the scope of this paper and helped us to improve the presentation clarity, and to Mrs. Agnieszka Pakuła for her help in correcting the text. 
Open Access This article is distributed under the terms of the Creative Commons Attribution 4.0 International License (http://creativecommons.org/licenses/by/4.0/), which permits unrestricted use, distribution, and reproduction in any medium, provided you give appropriate credit to the original author(s) and the source, provide a link to the Creative Commons license, and indicate if changes were made.

\section{References}

1. Ai ZT, Mak CM (2013) CFD simulation of flow and dispersion around an isolated building: effect of inhomogeneous ABL and near-wall treatment. Atmos Environ 77:568-578. doi:10.1016/j.atmosenv. 2013.05.034

2. Ai ZT, Mak CM (2015) Large-eddy simulation of flow and dispersion around an isolated building: analysis of influencing factors. Comput Fluids 118:89-100. doi:10.1016/j.compfluid.2015.06.006

3. Allwine KJ, Leach MJ, Stockham JS, Shinn JH, Hosker RP, Bowers JF, Pace JC (2004) Overview of Joint URBAN 2003-an atmospheric dispersion study in Oklahoma City. In: Proceedingsof the 84-th AMS Meeting, Seattle

4. Allwine KJ, Shinn JH, Streit GE, Clawson KL, Brown M (2002) Overview of URBAN 2000: a multiscale field study of dispersion through an urban environment. Bull Am Meteor Soc 83(4):521-536

5. Baker CJ (1980) The turbulent horseshoe vortex. J Wind Eng Ind Aerodyn 6(1-2):9-23. doi:10.1016/ 0167-6105(80)90018-5

6. Britter RE, Hanna SR (2003) Flow and dispersion in urban areas. Annu Rev Fluid Mech 35(1):469-496. doi:10.1146/annurev.fluid.35.101101.161147

7. Cermak JE (1958) Wind tunnel for the study of turbulence in the atmospheric surface layer. Tech. Rep. CER58-JEC42, Ft. Collins

8. Cermak JE (1979) Applications of wind tunnels to investigation of wind-engineering problems. AIAA J 17(7):679-690. doi:10.2514/3.61203

9. Chen F, Kusaka H, Bornstein R, Ching J, Grimmond CSB, Grossman-Clarke S, Loridan T, Manning KW, Martilli A, Miao S, Sailor D, Salamanca FP, Taha H, Tewari M, Wang X, Wyszogrodzki AA, Zhang C (2011) The integrated WRF/urban modelling system: development, evaluation, and applications to urban environmental problems. Int J Climatol 31(2):273-288. doi:10.1002/joc.2158

10. Chen L-W, Watson JG, Chow JC, Green MC, Inouye D, Dick K (2012) Wintertime particulate pollution episodes in an urban valley of the Western US: a case study. Atmos Chem Phys 12(21):10,051-10,064

11. Clark TL, Farley RD (1984) Severe downslope windstorm calculations in two and three spatial dimensions using anelastic interactive grid nesting: A possible mechanism for gustiness. J Atmos Sci 41(3):329-350

12. Clyne J, Mininni P, Norton A, Rast M (2007) Interactive desktop analysis of high resolution simulations: application to turbulent plume dynamics and current sheet formation. New J Phys 9(8):301

13. Cotter CS, Smolarkiewicz PK, Szczyrba IN (2002) A viscoelastic fluid model for brain injuries. Int J Numer Methods Fluids 40(1-2):303-311. doi:10.1002/fld.287

14. Davenport AG (1966) The treatment of wind loading on tall buildings. In: Coull A, Smith BS (eds) Proceedings of the symposium on tall buildings with particular reference to shear wall structures

15. Drazin PG (1961) On the steady flow of a fluid of variable density past an obstacle. Tellus 13(2):239-251

16. Fedorovich E, Kaiser R, Rau M, Plate E (1996) Wind tunnel study of turbulent flow structure in the convective boundary layer capped by a temperature inversion. J Atmos Sci 53(9):1273-1289

17. Fernando HJS, Lee SM, Anderson J, Princevac M, Pardyjak E, Grossman-Clarke S (2001) Urban fluid mechanics: air circulation and contaminant dispersion in cities. Environ Fluid Mech 1(1):107-164. doi:10.1023/A:1011504001479

18. Gartmann A, Müller MD, Parlow E, Vogt R (2012) Evaluation of numerical simulations of $\mathrm{CO}_{2}$ transport in a city block with field measurements. Environ Fluid Mech 12(2):185-200. doi:10.1007/ s10652-011-9226-z

19. Ghizaru M, Charbonneau P, Smolarkiewicz PK (2010) Magnetic cycles in global large-eddy simulations of solar convection. Astrophys J Lett 715(2):L133. doi:10.1088/2041-8205/715/2/L133

20. Gousseau P, Blocken B, Stathopoulos T, van Heijst GJF (2011) CFD simulation of near-field pollutant dispersion on a high-resolution grid: A case study by LES and RANS for a building group in downtown Montreal. Atmos Environ 45(2):428-438. doi:10.1016/j.atmosenv.2010.09.065

21. Gousseau P, Blocken B, van Heijst GJF (2013) Quality assessment of Large-Eddy simulation of wind flow around a high-rise building: validation and solution verification. Comput Fluids 79:120-133. doi:10.1016/j.compfluid.2013.03.006 
22. Grabowski WW, Smolarkiewicz PK (2002) A multiscale anelastic model for meteorological research. Mon Wea Rev 130(4):939-956. doi:10.1175/1520-0493(2002)130

23. Hanna SR, Brown MJ, Camelli FE, Chan ST, Coirier WJ, Kim S, Hansen OR, Huber AH, Reynolds RM (2006) Detailed simulations of atmospheric flow and dispersion in downtown Manhattan: an application of five computational fluid dynamics models. Bull Am Meteor Soc 87(12):1713-1726. doi:10.1175/ BAMS-87-12-1713

24. Hanna SR, White J, Zhou Y (2007) Observed winds, turbulence, and dispersion in built-up downtown areas of Oklahoma City and Manhattan. Bound Layer Meteorol 125(3):441-468. doi:10.1007/s10546007-9197-2

25. Hawthorne WR, Martin ME (1955) The effect of density gradient and shear on the flow over a hemisphere. Proc Roy Soc Lond A 232(1189):184-195. doi:10.1098/rspa.1955.0210

26. Hunt JCR (1971) A theory for the laminar wake of a two-dimensional body in a boundary layer. J Fluid Mech 49(01):159-178. doi:10.1017/S0022112071001988

27. Hunt JCR (1971) The effect of single buildings and structures. Philos Trans Roy Soc Lond A 269(1199):457-467. doi:10.1098/rsta.1971.0044

28. Iaccarino G, Ooi A, Durbin PA, Behnia M (2003) Reynolds averaged simulation of unsteady separated flow. Int J Heat Fluid Flow 24(2):147-156. doi:10.1016/S0142-727X(02)00210-2

29. Jensen M, Franck N (1963) Model scale tests in turbulent wind. Danish Technical Press

30. Kothari KM, Peterka JA, Meroney RN (1986) Perturbation analysis and measurements of building wakes in a stably stratified turbulent boundary layer. J Wind Eng Ind Aerodyn 25(1):49-74. doi:10. 1016/0167-6105(86)90104-2

31. Kothari KM, Peterka JA, Meroney RN (1979) Stably stratified building wakes. Tech. Rep. CER7879KMK-JAP-RNM65

32. Kukkonen J, Pohjola M, Sokhi RS, Luhana L, Kitwiroon N, Fragkou L, Rantamäki M, Berge E, Ødegaard V, Slørdal LH, Denby B, Finardi S (2005) Analysis and evaluation of selected local-scale PM10 air pollution episodes in four European cities: Helsinki, London, Milan and Oslo. Atmos Environ 39(15):2759-2773

33. Kuznetsova IN, Nakhaev MI, Shalygina IYu, Lezina EA (2008) Meteorological prerequisites of formation of severe wintertime air pollution episodes in Moscow. Russ Meteorol Hydrol 33(3):167-174

34. Li X-X, Britter RE, Norford LK (2015) Transport processes in and above two-dimensional urban street canyons under different stratification conditions: results from numerical simulation. Environ Fluid Mech 15(2):399-417. doi:10.1007/s10652-014-9347-2

35. Lipps FB, Hemler RS (1982) A scale analysis of deep moist convection and some related numerical calculations. J Atmos Sci 39:2192-2210

36. Lyra G. Über den Einfluß von Bodener hebungen auf die Strömung einer stabil geschichteten Atmosphäre. Beitr Phys Freien Atmos 26:197-206

37. Łobocki L (2013) Analysis of vertical turbulent heat flux limit in stable conditions with a local equilibrium, turbulence closure model. Bound Layer Meteorol 148(3):541-555. doi:10.1007/s10546-0139836-8

38. Malek E, Davis T, Martin RS, Silva PJ (2006) Meteorological and environmental aspects of one of the worst national air pollution episodes (January, 2004) in Logan, Cache Valley, Utah, USA. Atmos Res 79(2):108-122

39. Margolin, LG, Smolarkiewicz PK, Sorbjan Z. Large-eddy simulations of convective boundary layers using nonoscillatory differencing. Physica D 133:390-397

40. Meroney RN, Cermak JE, Yang BT (1975) Modeling of atmospheric transport and fumigation at shoreline sites. Bound Layer Meteorol 9(1):69-90. doi:10.1007/BF00232255

41. Mellor GL, Yamada T (1974) A hierarchy of turbulence closure models for planetary boundary layers. J Atmos Sci 31(7):1791-1806. doi:10.1175/1520-0469(1974)031

42. Mellor GL, Yamada T (1982) Development of a turbulence closure model for geophysical fluid problems. Rev Geophys Space Phys 20(4):851-875. doi:10.1029/RG020i004p00851

43. Mittal R, Iaccarino G (2005) Immersed boundary methods. Annu Rev Fluid Mech 37(1):239-261. doi:10.1146/annurev.fluid.37.061903.175743

44. Murakami S (1993) Comparison of various turbulence models applied to a bluff body. J Wind Eng Ind Aerodyn 46-47:21-36. doi:10.1016/0167-6105(93)90112-2

45. Murakami S (1998) Overview of turbulence models applied in CWE-1997. J Wind Eng Ind Aerodyn 74-76:1-24. doi:10.1016/S0167-6105(98)00004-X

46. Murakami S, Uehara K, Komine H (1979) Amplification of wind speed at ground level due to construction of high-rise building in urban area. J Wind Eng Ind Aerodyn 4(3-4):343-370. doi:10.1016/ 0167-6105(79)90012-6 
47. Nakanishi M, Niino H (2009) Development of an improved turbulence closure model for the atmospheric boundary layer. J Met Soc Jpn 87(5):895-912

48. Nappo CJ (2002) An introduction to atmospheric gravity waves : international geophysics series, vol. 85. Academic Press

49. Ogawa Y, Diosey PG, Uehara K, Ueda H (1981) A wind tunnel for studying the effects of thermal stratification in the atmosphere. Atmos Environ 15(5):807-821. doi:10.1016/0004-6981(81)90286-9

50. Ohya Y, Tatsuno M, Nakamura Y, Ueda H (1996) A thermally stratified wind tunnel for environmental flow studies. Atmos Environ 30(16):2881-2887. doi:10.1016/1352-2310(95)00256-1

51. The PanthaRhei Project info at the ECMWF website, http://www.ecmwf.int/en/research/projects/ pantarhei. Accessed 14 Feb 2016

52. Park S-B, Baik J-J, Han B-S (2015) Large-eddy simulation of turbulent flow in a densely built-up urban area. Environ Fluid Mech 15(2):235-250. doi:10.1007/s10652-013-9306-3

53. Peterka JA, Meroney RN, Kothari KM (1985) Wind flow patterns about buildings. J Wind Eng Ind Aerodyn 21(1):21-38. doi:10.1016/0167-6105(85)90031-5

54. Plate EJ, Cermak JE (1963) Micrometeorological Wind-Tunnel Facility. Tech. Rep. CER63EJP-JEC9, Ft. Collins

55. Prusa JM, Smolarkiewicz PK, Wyszogrodzki AA (2008) EULAG, a computational model for multiscale flows. Comput Fluids 37(9):1193-1207. doi:10.1016/j.compfluid.2007.12.001

56. Rau M, Bächlin W, Plate EJ (1991) Detailed design features of a new wind tunnel for studying the effects of thermal stratification. Atmos Environ 25(7):1257-1262. doi:10.1016/0960-1686(91)90236-Z

57. Rosa B, Kurowski M, Ziemiaski M (2011) Testing the anelastic nonhydrostatic model EULAG as a prospective dynamical core of a numerical weather prediction model. Part I: Dry benchmarks. Acta Geophys 59(6):12361266. doi:10.2478/s11600-011-0041-1

58. Rotach MW, Gryning S-E, Batchvarova E, Christen A, Vogt R (2004) Pollutant dispersion close to an urban surface-the BUBBLE tracer experiment. Meteorol Atmos Phys 87(1-3):39-56. doi:10.1007/ s00703-003-0060-9

59. Santos JM, Reis NC, Goulart EV, Mavroidis I (2009) Numerical simulation of flow and dispersion around an isolated cubical building: the effect of the atmospheric stratification. Atmos Environ 43:5484-5492. doi:10.1016/j.atmosenv.2009.07.020

60. Schatzmann M, Donat J, Hendel S, Krishan G (1995) Design of a low-cost stratified boundary-layer wind tunnel. J Wind Eng Ind Aerodyn 54-55:483-491. doi:10.1016/0167-6105(94)00061-H

61. Scorer RS (1949) Theory of waves in the lee of mountains. Q J Roy Meteor Soc 75(323):41-56

62. Scorer RS (1978) Environmental Aerodynamics. Wiley, New York

63. Sheppard PA (1956) Airflow over mountains. Q J Roy Meteor Soc 82(354):528-529

64. Smith RB (1980) Linear theory of stratified hydrostatic flow past an isolated mountain. Tellus 32(4):348-364

65. Smolarkiewicz PK, Charbonneau P (2013) EULAG, a computational model for multiscale flows: an MHD extension. J Comput Phys 236:608-623. doi:10.1016/j.jcp.2012.11.008

66. Smolarkiewicz PK, Doernbrack A (2008) Conservative integrals of adiabatic Durran equations. Int J Numer Methods Fluids 56:1529-1534

67. Smolarkiewicz PK (2006) Multidimensional positive definite advection transport algorithm: an overview. Int J Numer Methods Fluids 50:112344

68. Smolarkiewicz PK, Margolin LG (1994) Variational solver for elliptic problems in atmospheric flows. Appl Math Comput Sci 4:527551

69. Smolarkiewicz PK, Margolin LG, Wyszogrodzki AA (2001) A class of nonhydrostatic global models. J Atmos Sci 58(4):349-364

70. Smolarkiewicz PK, Rotunno R (1989) Low Froude number flow past three-dimensional obstacles. Part I: baroclinically generated lee vortices. J Atmos Sci 46(8):1154-1164

71. Smolarkiewicz PK, Sharman R, Weil J, Perry SG, Heist D, Bowker G (2007) Building resolving largeeddy simulations and comparison with wind tunnel experiments. J Comput Phys 227(1):633-653. doi:10.1016/j.jcp.2007.08.005

72. Smolarkiewicz PK, Winter CL (2010) Pores resolving simulation of Darcy flows. J Comput Phys 229. doi:10.1016/j.jcp.2009.12.031

73. Sorbjan Z (1996) Numerical study of penetrative and solid lid nonpenetrative convective boundary layers. J Atmos Sci 53:101112

74. Tamura T, Nozawa K, Kondo K (2008) AIJ guide for numerical prediction of wind loads on buildings. J Wind Eng Ind Aerodyn 96(10-11):1974-1984. doi:10.1016/j.jweia.2008.02.020

75. Taylor PA, Delage Y (1971) A note on finite-difference schemes for the surface and planetary boundary layers. Bound Layer Meteorol 2(1):108-121

76. The NCAR Command Language (version 6.3.0) [software] (2015). doi:10.5065/D6WD3XH5 
77. The Palace of Culture and Science website. http://www.pkin.pl/en. Accessed 21 Sept 2015

78. Tomas JM, Pourquie MJBM, Jonker HJJ (2015) The influence of an obstacle on flow and pollutant dispersion in neutral and stable boundary layers. Atmos Environ 113:236-246

79. Tomas JM, Pourquie MJBM, Jonker HJJ (2016) Stable stratification effects on flow and pollutant dispersion in boundary layers entering a generic urban environment. Bound Layer Meteorol 159(2):221-239

80. Tominaga Y, Mochida A, Murakami S, Sawaki S (2008) Comparison of various revised $k-\varepsilon$ models and LES applied to flow around a high-rise building model with 1:1:2 shape placed within the surface boundary layer. J Wind Eng Ind Aerodyn 96(4):389-411. doi:10.1016/j.jweia.2008.01.004

81. Tominaga Y, Mochida A, Yoshie R, Kataoka H, Nozu T, Yoshikawa M, Shirasawa T (2008) AIJ guidelines for practical applications of CFD to pedestrian wind environment around buildings. J Wind Eng Ind Aerodyn 96(10-11):1749-1761. doi:10.1016/j.jweia.2008.02.058

82. Uehara K, Murakami S, Oikawa S, Wakamatsu S (2000) Wind tunnel experiments on how thermal stratification affects flow in and above urban street canyons. Atmos Environ 34(10):1553-1562. doi:10. 1016/S1352-2310(99)00410-0

83. Warn-Varnas A, Hawkins J, Smolarkiewicz PK, Chin-Bing SA, King D, Hallock Z (2007) Solitary wave effects north of Strait of Messina. Ocean Model 18:97-121

84. Warszawa 3D—model przestrzenny miasta. (Fotokart web page). http://www.zenit.szczecin.pl/index. php?page=realizacje \&subpage=warszawa1. Accessed 13 June 2016

85. Wedi NP, Smolarkiewicz PK (2005) Laboratory for internal gravity-wave dynamics: the numerical equivalent to the quasi-biennial oscillation (QBO) analogue. Int J Numer Methods Fluids 47:13691374

86. Woo HGC, Peterka JA, Cermak JE (1977) Wind tunnel measurements in the wakes of structures. Tech. rep

87. Wyszogrodzki, AA, Smolarkiewicz, PK (2009) Building resolving large-eddy simulations (LES) with EULAG. In: Academy colloquium on immersed boundary methods: current status and future research directions, 15-17 June 2009, Academy Building, Amsterdam

88. Wyszogrodzki AA, Shiguang M, Chen F (2012) Evaluation of the coupling between mesoscale-WRF and LES-EULAG models for simulation fine-scale urban dispersion. Atmos Res 118:324-345. doi:10. 1016/j.atmosres.2012.07.023

89. Yan BW, Li QS (2015) Inflow turbulence generation methods with large eddy simulation for wind effects on tall buildings. Comput Fluids 116:158-175. doi:10.1016/j.compfluid.2015.04.020

90. Zhang YQ, Arya SPS, Snyder WH (1996) A comparison of numerical and physical modeling of stable atmospheric flow and dispersion around a cubical building. Atmos Environ 30(8):1327-1345. doi:10.1016/1352-2310(95)00326-6 